\title{
Coherently Aligned Porphyrin-Appended Polynorbornenes
}

\author{
Hsian-Wen Wang, ${ }^{[a]}$ Zhi-Chang Liu, ${ }^{[b]}$ Chih-Hsien Chen, ${ }^{[a]}$ Tsong-Shin Lim, ${ }^{[c]}$ \\ Wunshain Fann ${ }^{\dagger},{ }^{[d]}$ Chih-Gang Chao, ${ }^{[a]}$ Jian-Yuan Yu, ${ }^{[a]}$ Shern-Long Lee, ${ }^{[a]}$ \\ Chun-hsien Chen, ${ }^{[a]}$ Shou-Ling Huang, ${ }^{[a]}$ and Tien-Yau Luh*[a, b]
}

\author{
Dedicated to Professor Armin de Meijere on the occasion of his 70th birthday
}

\begin{abstract}
Six different kinds of coherently aligned porphyrin-appended polynorbornenes derived from 5,6-endofused $\quad N$-arylpyrrolidenonorbornenes have been synthesized. $\pi-\pi$ interactions between the pendant groups are essential for dictating the photophysical properties of the polymers and the mechanism for the stereoselective formation of polymers. Splitting of the Soret band of polymers $\mathbf{2 a}-\mathbf{c}$, which
\end{abstract}

have alkyl-substituted porphyrin pendant groups, suggests strong exciton coupling between chromophores. No splitting of the Soret band is observed for polymers $2 \mathbf{d}-\mathbf{f}$, which have tetraaryl

Keywords: Arylpyrrolidenes photophysics - polynorbornene • porphyrinoids $\cdot$ ring-opening polymerization substituents on the porphyrin moiety. Significant fluorescence quenching is found in polymers $\mathbf{2 a}-\mathbf{e}$, whereas only slightly reduced quantum yield is observed for $\mathbf{2}$ f. Time-resolved fluorescence measurements also indicate a similar trend. The AFM image of $\mathbf{2 d}$ on graphite shows aggregation to form a two-dimensional, ordered pattern.

\section{Introduction}

The photosynthetic system of purple bacteria has been shown to contain a supramolecular array of chlorophyll moi-

[a] H.-W. Wang, Dr. C.-H. Chen, C.-G. Chao, J.-Y. Yu, S.-L. Lee, Prof. C.-h. Chen, S.-L. Huang, Prof. T.-Y. Luh

Department of Chemistry

National Taiwan University

Taipei, 106 (Taiwan)

Fax: (+886)2-2364-4971

E-mail: tyluh@ntu.edu.tw

[b] Z.-C. Liu, Prof. T.-Y. Luh

Shanghai Institute of Organic Chemistry

Chinese Academy of Sciences

354 Fenglin Lu, Shanghai 200032 (China)

[c] Prof. T.-S. Lim

Department of Physics

Tung Hai University

Taichung, 407 (Taiwan)

[d] Prof. W. Fann

Institute of Atomic and Molecular Science

Academia Sinica

Taipei, 106 (Taiwan)

[†] Deceased.

Supporting information for this article is available on the WWW under http://dx.doi.org/10.1002/chem.200900195. eties and pigments within protein matrices, in which efficient capture of solar energy and electron transfer take place. ${ }^{[1]}$ Relative orientations and distances between chromophores may be crucial for these important, efficient, and ultrafast photoinduced processes. Numerous model systems with different cofacial alignments of porphyrin derivatives have been designed to mimic this important biological process. ${ }^{[2]}$ For example, cofacial bisporphyrin dyads, ${ }^{[2]]}$ multiple deck oligoporphyrins, ${ }^{[3]}$ multiporphyrinic dendrimers, ${ }^{[4]}$ and porphyrin-appended macromolecules ${ }^{[5]}$ have been extensively examined for through-space singlet and/or triplet energy transfers. A molecular assembly based on derivatized polystyrenes with pendant polypyridine complexes of $\mathrm{Ru}^{\mathrm{II}}$ has been used to mimic both the light-harvesting and energyconversion steps of photosynthesis. ${ }^{[6]}$ More rigid polynorbornenes with electroactive pendants have also been employed for photoinduced electron transfer investigations. ${ }^{[7]}$ The use of tetraphenylporphyrin pendant groups in a norbornenebased block copolymer has been briefly explored for a possible molecular switch device. ${ }^{[8]}$ It has recently been shown that polynorbornenes with $\mathrm{N}$-aryl-substituted 5,6-endo-fused pyrrolidine, obtained by ring-opening metathesis polymerization (ROMP) of the corresponding norbornene monomers catalyzed by the Grubbs I catalyst, may have homogeneous stereochemistry in which all pendants align coherently in 
the same direction. ${ }^{[9]}$ In addition, the double bonds in these polymers were predominantly, if not exclusively, trans. The space occupied by each of the monomeric units in these polymers is about $5.5 \AA^{\left[{ }^{[9]}\right.}$ This strategy has been employed for the design and synthesis of double-stranded polybisnorbornenes. ${ }^{[10]}$ Electrochemical and magnetic investigations of these double-stranded polymers showed that there are strong interactions between the ferrocene linkers due to their close proximity. ${ }^{[11]}$ It is envisaged that the incorporation of porphyrin pendants in polynorbornenes would provide an intriguing model in which all porphyrin moieties would be aligned in a similar direction and that interactions between these pendant macrocyclic aromatic rings might offer intriguing photophysical behavior. Herein we wish to report the synthesis and photophysical properties of porphyrin-appended polynorbornenes.

\section{Results and Discussion}

Synthesis: The porphyrin moieties in norbornene monomers 1a-f were prepared by using the Lindsey procedure (Schemes 1 and 2) and the details are described in the Experimental Section. ${ }^{[12]}$ Polymerization of $\mathbf{1 a}-\mathbf{f}$ in the presence of the Grubbs I catalyst afforded the corresponding dark purple polymers $\mathbf{2 a - f}$ (Scheme 2) in good yields. The
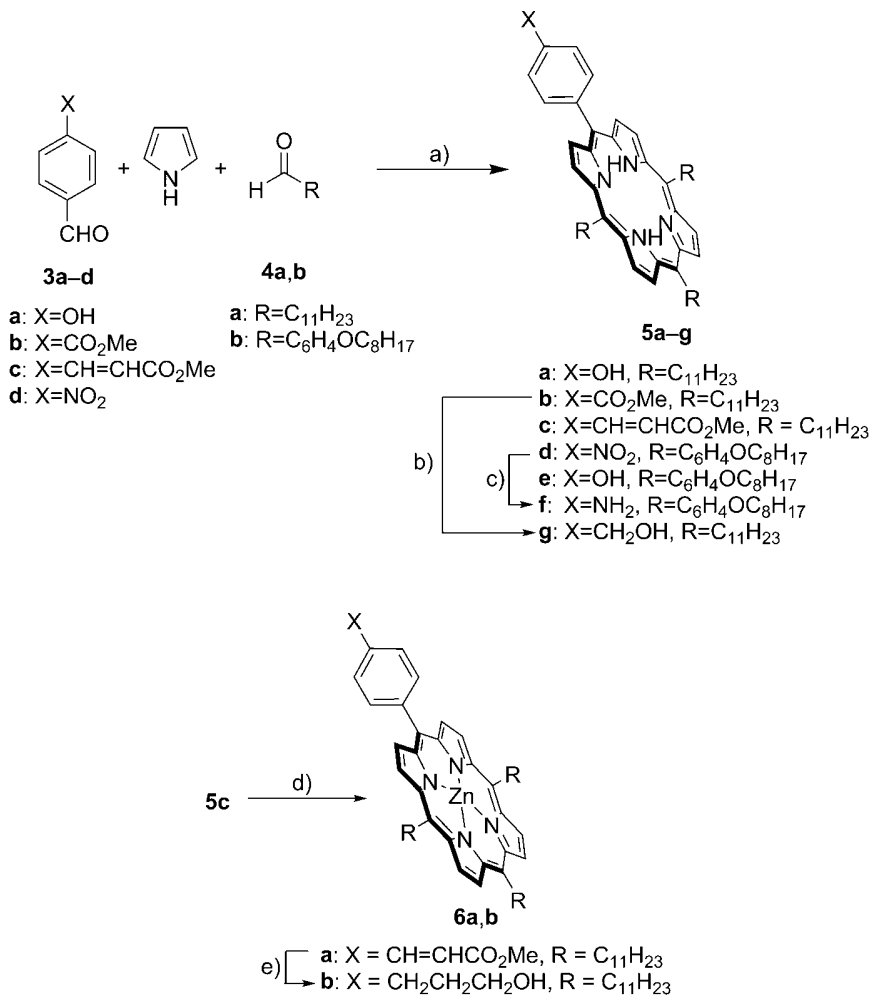

Scheme 1 . Synthesis of substituted porphyrins 5 : a) $\mathrm{BF}_{3} \cdot \mathrm{OEt}_{2}$ or TFA (trifluoroacetic acid), then DDQ (2,3-dichloro-5,6-dicyano-1,4-benzoquinone), then $\mathrm{NEt}_{3}$, RT; b) DIBAL-H (diisobutylaluminum hydride); c) $\mathrm{SnCl}_{2} \cdot 2 \mathrm{H}_{2} \mathrm{O}$, concd $\mathrm{HCl}$; d) $\mathrm{Zn}(\mathrm{OAc})_{2} \cdot 2 \mathrm{H}_{2} \mathrm{O}$; e) $\mathrm{H}_{2}, \mathrm{Pd} / \mathrm{C}$, then DIBAL-H.
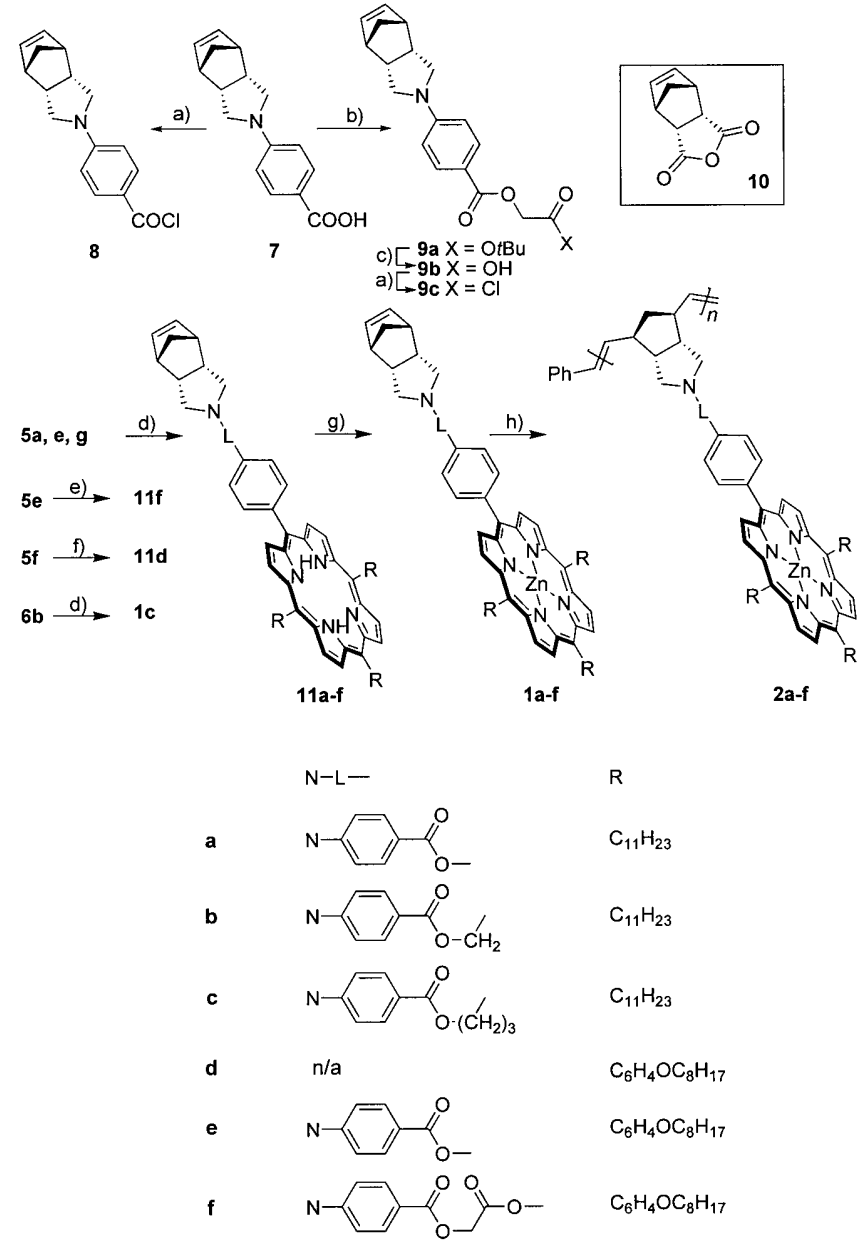

Scheme 2. Synthesis of $\mathbf{1}$ and $\mathbf{2}(\mathrm{n} / \mathrm{a}=$ no linker $)$. a) $(\mathrm{COCl})_{2}$; b) $\mathrm{Cs}_{2} \mathrm{CO}_{3}$, $\mathrm{BrCH}_{2} \mathrm{CO}_{2} \mathrm{Bu}$; c) TFA; d) $8, \mathrm{NEt}_{3}$, DMAP (4-dimethylaminopyridine); e) 9, $\mathrm{NEt}_{3}, \mathrm{DMAP}$; f) Compound 10, then LAH (lithiumaluminum hydride); g) $\left.\mathrm{Zn}(\mathrm{OAc})_{2} \cdot 2 \mathrm{H}_{2} \mathrm{O} ; \mathrm{h}\right)\left[\left(\mathrm{Cy}_{3}\right)_{2} \mathrm{Cl}_{2} \mathrm{Ru}=\mathrm{CHPh}\right]$.

${ }^{13} \mathrm{C}$ NMR spectra of $\mathbf{2 a - f}$ were similar to those of corresponding monomers $1 \mathbf{a}-\mathbf{g}$ and consistent with the structures.

${ }^{1}$ H NMR spectra: The partial ${ }^{1} \mathrm{H}$ NMR spectra of the aromatic regions of $\mathbf{2} \mathbf{a}-\mathbf{f}$ are compared with those of $\mathbf{1 a}-\mathbf{f}$ in Figure 1. Besides severe line broadening, ${ }^{[13]}$ the signals for the protons on porphyrin moieties in $\mathbf{2 a - f}$ were shifted upfield compared with those of the corresponding monomers 1a-c due to anisotropic shielding by the neighboring porphyrin moieties. Similar behavior was found in cofacial multiple deck oligoporphyrins ${ }^{[3]}$ and related systems. ${ }^{[14]}$ These results suggest that the neighboring porphyrin pendants in 2 a-c may be in close proximity and, therefore, these chromophores may align coherently in a similar direction.

As can be seen from Figure 1, the signals for the $\beta$ protons on the porphyrin rings in $\mathbf{2} \mathbf{a}-\mathbf{c}$ were more affected than those in $\mathbf{2 d - f}$. These results suggest that the neighboring porphyrin chromophores would be in closer proximal relationships for $\mathbf{2 a - c}$ than those for $\mathbf{2 d - f}$. It is worth noting that polymers $\mathbf{2 d - f}$ have aryl substituents at the other mesopositions of the porphyrin skeleton whereas $\mathbf{2 a - c}$ have alkyl 


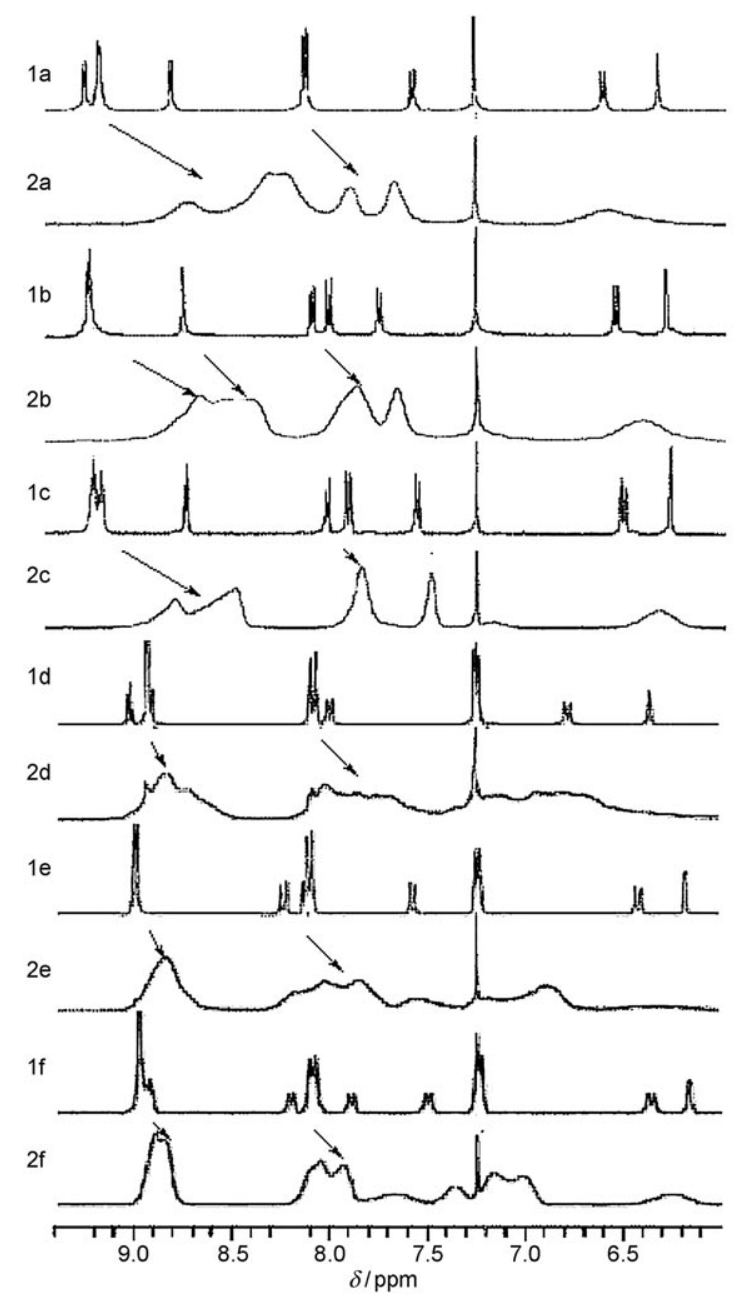

Figure 1. Partial ${ }^{1} \mathrm{H}$ NMR spectra (aromatic region) of $\mathbf{1 a - f}$ and $\mathbf{2 a - f}$ in $\mathrm{CDCl}_{3}$.

substituents at the same positions. Tetraphenylporphyrin is known to be nonplanar. ${ }^{[15]}$ As mentioned in the Introduction Section, the spacing occupied by each of the monomeric units in 2 would be around $5.5 \AA$. Steric repulsion between neighboring pendant groups in $\mathbf{2 d - f}$ might be expected if they were in more eclipsed conformations. It seems likely that the porphyrin pendants in $\mathbf{2 d - f}$ might be more staggered than those in $\mathbf{2 a - c}$. These characteristics might also be reflected in their photophysical properties as discussed below.

Tapping-mode atomic force microscopy (TMAFM) images: TMAFM was carried out to morphologically assess the cofacial stacking of the appended porphyrins. To facilitate the imaging, the sample was subjected to shear treatment ${ }^{[16]} \mathrm{im}$ mediately after it was dropcast on the freshly cleaved surface of highly ordered pyrolytic graphite. A typical image of polymer $\mathbf{2 d}$, which is a prototype for this series of polynorbornenes, is displayed in Figure 2 and shows a long-range ordered pattern with stripes significantly longer than the apparent length of $\mathbf{2 d}$. Such an assembly is common for

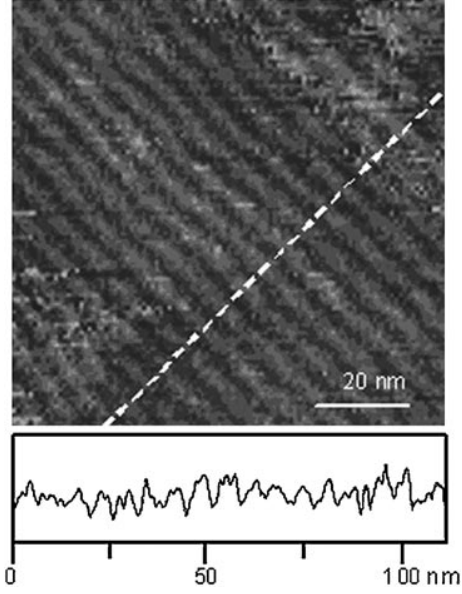

Figure 2. Top: TMAFM image of $\mathbf{2 d}$ on highly ordered pyrolytic graphite. Bottom: The section profile of the dash line showing the spacing of about $5 \mathrm{~nm}$. Imaging conditions: $257 \mathrm{kHz}$ for the drive frequency of the tip.

double-stranded polybisnorbornenes ${ }^{[10,11]}$ and is ascribed to intermolecular interactions, such as $\pi-\pi$ stacking between terminal groups along the longitudinal dimension and van der Waal interactions between the polymeric backbones and pendant groups in the horizontal direction. The aligned stripes have a uniform width of around $5 \mathrm{~nm}$. The uniformity and the dimension suggest the porphyrin pendants are cofacially attached to the bisnorbornene backbone.

Photophysical properties: The absorption spectra of 1a-f and $2 \mathbf{a}-\mathbf{f}$ in $\mathrm{CH}_{2} \mathrm{Cl}_{2}$ are shown in Figure 3 and selected photophysical properties are summarized in Table 1 . It is worth noting that the Soret band of polymers $\mathbf{2 a - c}$ split into two bands, which indicates that there may be significant exciton coupling between neighboring porphyrin chromophores even though the distance between these neighboring pendant moieties would be around $5.5 \AA$. In addition, the relative intensities of the shorter wavelength absorption versus the longer wavelength absorption in the split Soret band decreased as the number of methylene moieties in the linkers of $\mathbf{2} \mathbf{a}-\mathbf{c}$ increased. The incorporation of methylene groups in the pendants may increase its flexibility and the exciton coupling between meighboring chromophores may be affected.

It is known that intramolecular interactions between two porphyrin moieties leading to the splitting of the Soret band may depend on the distance and dihedral angle between the two chromophores. ${ }^{[8,17,18]}$ In general, the exciton interaction between two porphyrins is relatively weak if the face-to-face distance between porphyrins in cofacial bisporphyrins is more than $5 \AA{ }^{[19]}$ The $5.5 \AA$ distance between the cofacial porphyrin chromophores in $\mathbf{2} \mathbf{a}-\mathbf{c}$ would be rather large to exhibit exciton coupling. ${ }^{[20]}$ Slight flexibility in the linkers of 2a-c might result in a closer face-to-face distance between the neighboring porphyrin moieties or adjust the dihedral angle between two transition dipoles and lead to exciton coupling. 

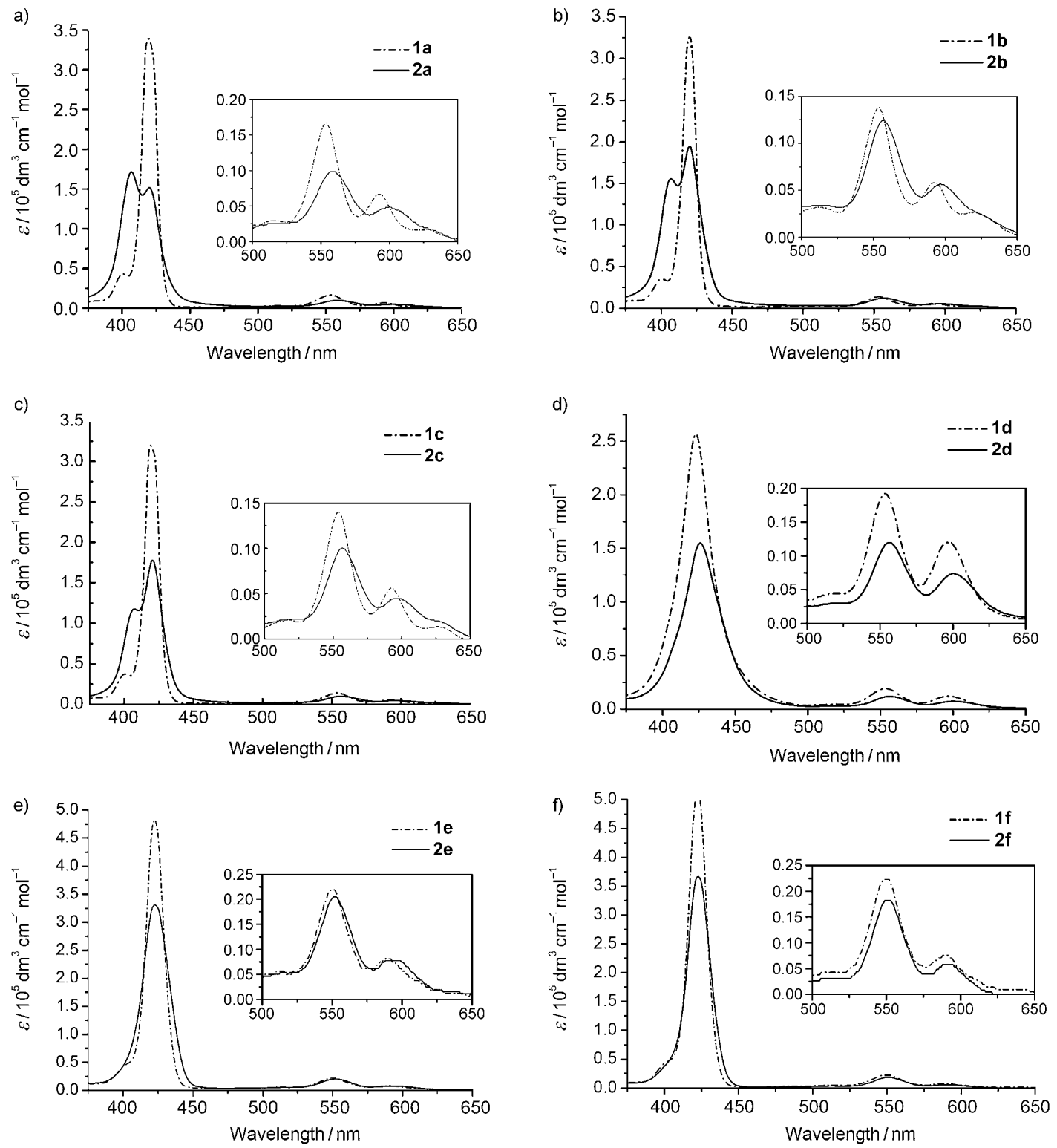

Figure 3. Absorption spectra of $\mathbf{1} \mathbf{a}-\mathbf{f}$ and $\mathbf{2} \mathbf{a}-\mathbf{f}$ in $\mathrm{CH}_{2} \mathrm{Cl}_{2}$.

Table 1. UV/Vis absorption and fluorescent spectral data for $\mathbf{1}$ and $\mathbf{2}$ in $\mathrm{CH}_{2} \mathrm{Cl}_{2}$.

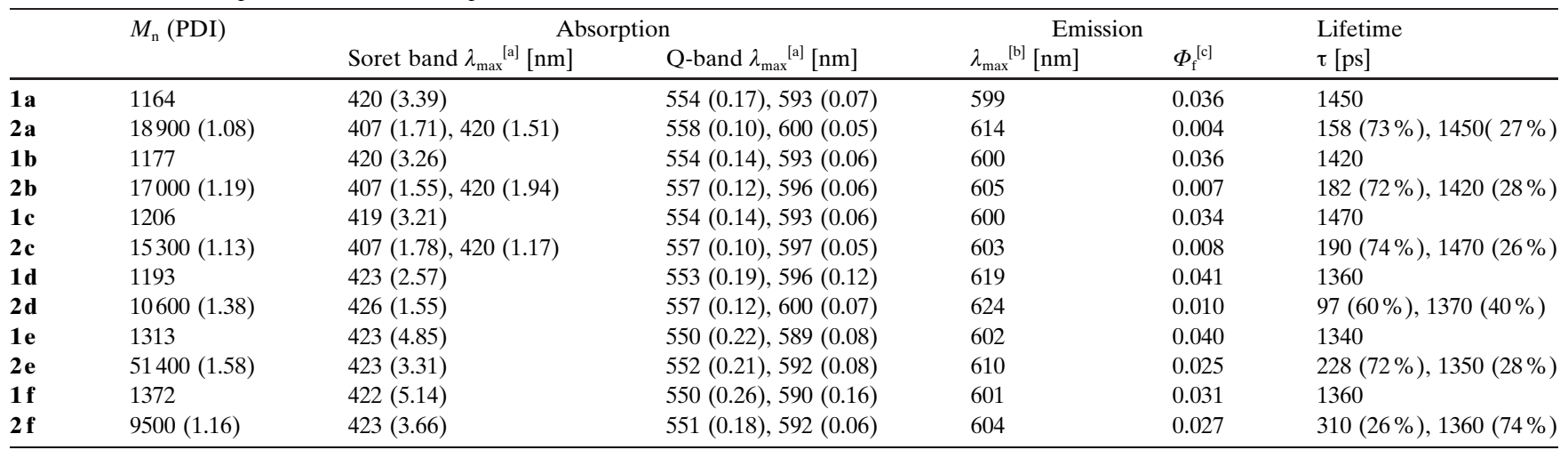

[a] The molar extinction coefficient $\left(10^{5} \mathrm{M}^{-1} \mathrm{~cm}^{-1}\right)$ based on the molecular weight of the monomeric unit is shown in parentheses. [b] Excitation at $\lambda_{\text {max }}$ around $550 \mathrm{~nm}$. [c] The quantum yield for ZnTPP in toluene, used as a reference, is 0.033 (see ref. [12b]). 
As shown in Figure 3, the Soret bands of $\mathbf{2} \mathbf{d}-\mathbf{f}$ showed essentially no change compared with those of the corresponding monomers 1d-f. Presumably, little aggregation resulting in exciton coupling may take place for $\mathbf{2} \mathbf{d}-\mathbf{f}$ because of the presence of four aryl substituents on the porphyrin ring. ${ }^{[15]}$

The fluorescence quantum yields of polymers $\mathbf{2} \mathbf{a}-\mathbf{c}$ were significantly reduced compared with those of the corresponding monomers 1a-c, presumably due to self quenching. It is interesting to note that the reduction in quantum yield for polymers $\mathbf{2 d - f}$ compared with those of the corresponding monomers $\mathbf{1 d}-\mathbf{f}$ were, relatively speaking, smaller than for those of alkyl-substituted analogues $\mathbf{2 a - c}$. These results appear to be consistent with other photophysical data and the NMR results described above.

Time-resolved fluorescence spectroscopy: Time-resolved fluorescence decays for both monomers $\mathbf{1 a}-\mathbf{f}$ and polymers $2 \mathbf{a}-\mathbf{f}$ in $\mathrm{CH}_{2} \mathrm{Cl}_{2}$ were measured. Using two-exponential fittings, the fluorescence lifetimes are also summarized in Table 1.

The longer lifetimes for $\mathbf{2} \mathbf{a}-\mathbf{f}$ were comparable with those of corresponding monomers $\mathbf{1 a}-\mathbf{f}$. The major route for the fluorescence decay $(60-73 \%)$ occurred via the shorter lifetime path, presumably because of self-quenching between neighboring porphyrin moieties in $\mathbf{2 a}-\mathbf{f}$. As can be seen from Table 1, these lifetimes fall within the same range as those obtained from related cofacial porphyrin dyads. ${ }^{[21]}$ Similar quenching behavior can also be found in chlorophyll- and dye-based systems. ${ }^{[22]}$ These results again suggest that all porphyrin pendants are coherently aligned in a similar direction.

Importance of the endo-fused $\boldsymbol{N}$-arylpyrrolidine moiety: In spite of the large porphyrin pendant groups in $\mathbf{2}$, the stereoselectivity (trans double bonds) and syn orientation of the pendants appeared to be similar to those of simple aryl-substituted 5,6-endo-pyrrolidine-fused polynorbornenes. ${ }^{[9]}$ It seems likely that $\pi-\pi$ interactions would be essential for controlling the stereochemistry of the polymerization during the course of the ROMP process. To test the validity of this hypothesis, ROMP of $\mathbf{1 2}$ was carried out under the usual conditions to give $\mathbf{1 3}$ containing a mixture of cis and trans double bonds (21:79) in $81 \%$ yield.
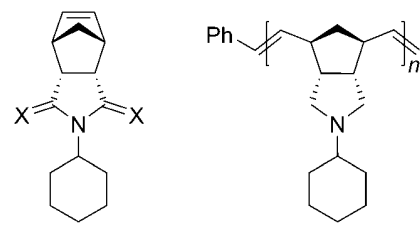

12a $(X=O)$ $12 b(X=H, H)$ 13

\section{Conclusions}

We have demonstrated direct evidence to show that porphyrin-appended polynorbornenes derived from 5,6-endo-fused
$N$-aryl-pyrrolidenonorbornenes may have coherently aligned pendant groups that exhibit exciton coupling and fluorescence quenching in the absorption and emission profiles. $\pi-$ $\pi$ interactions between the pendant groups were essential to dictate the photophysical properties of the polymers and the mechanism for the stereoselective formation of $\mathbf{2}$. Our results suggest that the presence of the $N$-aryl group is essential for dictating the stereoselectivity of the ROMP of $\mathbf{1}$. Because of the close proximity between the porphyrin moieties, these polymers may provide a versatile model system for studying light harvesting and photoinduced electron transfer processes.

\section{Experimental Section}

General: High-resolution mass spectrometric measurements were obtained by using a Jeol-JMS-700 mass spectrometer using the FAB method with a 3-nitrobenzyl alcohol matrix. Gel permeation chromatography (GPC) was performed by using a Waters GPC machine with an isocratic HPLC pump (1515) and a refractive index detector (2414). THF was used as the eluent (flow rate $=1.0 \mathrm{~mL} \mathrm{~min}^{-1}$ ). Waters Styragel HR2, HR3, and HR4 columns $(7.8 \times 300 \mathrm{~mm})$ were employed for determining the relative molecular weight with polystyrene as the standard $\left(M_{\mathrm{n}}\right.$ values ranged from 375 to $\left.3.5 \times 10^{6}\right)$. Absorption spectra were measured by using a Hitachi U-3310 spectrophotometer and emission spectra were recorded by using a Hitachi F-4500 fluorescence spectrophotometer. Quantum yields were obtained with $\mathrm{ZnTPP}$ in toluene as the reference $(\Phi=0.033)$.

21H,23H-5-(4-Hydroxyphenyl)-10,15,20-tris(undecyl)porphyrin $\quad$ (5a): TFA $\left(1.5 \mathrm{~mL}, 10^{-2} \mathrm{M}\right)$ was added slowly to a mixture of $\mathbf{3 a}(1.22 \mathrm{~g}$, $10 \mathrm{mmol}), 4 \mathrm{a}(6.01 \mathrm{~g}, 30 \mathrm{mmol})$, and pyrrole $(2.79 \mathrm{~mL}, 40 \mathrm{mmol})$ in $\mathrm{CH}_{2} \mathrm{Cl}_{2}(2 \mathrm{~L})$, then the mixture was stirred for $2 \mathrm{~h}$ before being quenched by the addition of DDQ $(6.81 \mathrm{~g}, 30 \mathrm{mmol})$. After stirring for $1 \mathrm{~h}$ at RT, $\mathrm{NEt}_{3}(5 \mathrm{~mL})$ was added to neutralize TFA. The solvent was removed in vacuo and the residue was purified by column chromatography on silica gel (eluent $\mathrm{CH}_{2} \mathrm{Cl}_{2}$ ) to give $\mathbf{5 a}$ as a purple solid $(650 \mathrm{mg}, 8 \%$ ). M.p. 87$88^{\circ} \mathrm{C} ;{ }^{1} \mathrm{H}$ NMR $\left(400 \mathrm{MHz}, \mathrm{CDCl}_{3}\right): \delta=-2.65(\mathrm{~s}, 2 \mathrm{H} ; \mathrm{NH}), 0.87-0.92(\mathrm{~m}$, $\left.9 \mathrm{H} ; \mathrm{CH}_{3}\right), 1.17-1.45\left(\mathrm{~m}, 36 \mathrm{H} ; \mathrm{CH}_{2}\right), 1.48-1.56\left(\mathrm{~m}, 6 \mathrm{H} ; \mathrm{CH}_{2}\right), 1.75-1.88$ $\left(\mathrm{m}, 6 \mathrm{H} ; \mathrm{CH}_{2}\right), 2.47-2.58\left(\mathrm{~m}, 6 \mathrm{H} ; \mathrm{CH}_{2}\right), 4.90-5.00\left(\mathrm{~m}, 6 \mathrm{H} ; \mathrm{CH}_{2}\right), 7.11(\mathrm{~d}$, ${ }^{3} J(\mathrm{H}, \mathrm{H})=8.1 \mathrm{~Hz}, 2 \mathrm{H}$; ArH $), 7.99\left(\mathrm{~d},{ }^{3} J(\mathrm{H}, \mathrm{H})=8.1 \mathrm{~Hz}, 2 \mathrm{H} ; \mathrm{ArH}\right), 8.83$ $\left(\mathrm{d},{ }^{3} J(\mathrm{H}, \mathrm{H})=4.7 \mathrm{~Hz}, 2 \mathrm{H}\right), 9.38\left(\mathrm{~d},{ }^{3} J(\mathrm{H}, \mathrm{H})=4.7 \mathrm{~Hz}, 2 \mathrm{H}\right), 9.49\left(\mathrm{~d},{ }^{3} J-\right.$ $(\mathrm{H}, \mathrm{H})=4.7 \mathrm{~Hz}, 2 \mathrm{H}), 9.52 \mathrm{ppm}\left(\mathrm{d},{ }^{3} J(\mathrm{H}, \mathrm{H})=4.7 \mathrm{~Hz}, 2 \mathrm{H}\right) ;{ }^{13} \mathrm{C} \mathrm{NMR}$ $\left(100 \mathrm{MHz}, \mathrm{CDCl}_{3}\right): \delta=14.1,22.7,29.3,29.4,29.64,29.66,29.71,29.73$, $30.6,30.7,31.9,35.4,35.8,38.7,38.9,113.5,117.7,119.1,119.3,127.9$, $128.2,135.1,135.5,136.3,155.2 \mathrm{ppm} ;$ IR $(\mathrm{KBr}): \tilde{v}=3383,3318,3129$, 2954, 2923, 2852, 1609, 1513, 1479, 1467, 1353, 1264, 1238, 964, 913, $791 \mathrm{~cm}^{-1}$; HRMS (FAB): $m / z$ calcd for $\mathrm{C}_{59} \mathrm{H}_{85} \mathrm{~N}_{4} \mathrm{O}: 865.6720\left[M^{+}+\mathrm{H}\right]$; found: 865.6723 .

21H,23H-5-\{4-[2-(Methoxycarbonyl)-1-vinyl]phenyl)-10,15,20-tris-

(undecyl)porphyrin $(\mathbf{5 c})$ : Boron trifluoride etherate $(0.8 \mathrm{~mL})$ was added slowly to a solution of $\mathbf{3 c}(1.00 \mathrm{~g}, 5.26 \mathrm{mmol}), \mathbf{4 a}(3.16 \mathrm{~g}, 15.78 \mathrm{~mol})$, and pyrrole $(1.5 \mathrm{~mL}, 21.03 \mathrm{mmol})$ in dry $\mathrm{CH}_{2} \mathrm{Cl}_{2}(2 \mathrm{~L})$ under nitrogen and the mixture was stirred at RT for $1 \mathrm{~h}$. DDQ $(7.16 \mathrm{~g}, 31.54 \mathrm{mmol})$ was added and the mixture was stirred at RT for $1 \mathrm{~h}$. The reaction mixture was neutralized with $\mathrm{NEt}_{3}(5 \mathrm{~mL})$ and the solvent was evaporated in vacuo. The residue was purified by column chromatography on silica gel (eluent hexane $\left./ \mathrm{CH}_{2} \mathrm{Cl}_{2} 1: 1\right)$ to give $5 \mathbf{c}(0.41 \mathrm{~g}, 8 \%)$. M.p. $56-57{ }^{\circ} \mathrm{C} ;{ }^{1} \mathrm{H}$ NMR $\left(400 \mathrm{MHz}, \mathrm{CDCl}_{3}\right): \delta=-2.65(\mathrm{~s}, 2 \mathrm{H}), 0.85-0.92(\mathrm{~m}, 9 \mathrm{H}), 1.27-1.33$ (brs, $36 \mathrm{H}), 1.50-1.56(\mathrm{~m}, 6 \mathrm{H}), 1.76-1.81(\mathrm{~m}, 6 \mathrm{H}), 2.49-2.53(\mathrm{~m}, 6 \mathrm{H}), 3.94(\mathrm{~s}$, $3 \mathrm{H}), 4.90-4.99(\mathrm{~m}, 6 \mathrm{H}), 6.76\left(\mathrm{~d},{ }^{3} J(\mathrm{H}, \mathrm{H})=16.1 \mathrm{~Hz}, 1 \mathrm{H}\right), 7.90\left(\mathrm{~d},{ }^{3} J-\right.$ $(\mathrm{H}, \mathrm{H})=8.1 \mathrm{~Hz}, 2 \mathrm{H}), 8.06\left(\mathrm{~d},{ }^{3} J(\mathrm{H}, \mathrm{H})=16.1 \mathrm{~Hz}, 1 \mathrm{H}\right), 8.19\left(\mathrm{~d},{ }^{3} J(\mathrm{H}, \mathrm{H})=\right.$ $8.1 \mathrm{~Hz}, 2 \mathrm{H}), 8.79\left(\mathrm{~d},{ }^{3} J(\mathrm{H}, \mathrm{H})=4.8 \mathrm{~Hz}, 2 \mathrm{H}\right), 9.39\left(\mathrm{~d},{ }^{3} J(\mathrm{H}, \mathrm{H})=4.8 \mathrm{~Hz}\right.$, $2 \mathrm{H}), 9.50\left(\mathrm{~d},{ }^{3} J(\mathrm{H}, \mathrm{H})=4.8 \mathrm{~Hz}, 2 \mathrm{H}\right), 9.53 \mathrm{ppm}\left(\mathrm{d},{ }^{3} J(\mathrm{H}, \mathrm{H})=4.8 \mathrm{~Hz}, 2 \mathrm{H}\right)$; ${ }^{13} \mathrm{C} \mathrm{NMR}\left(100 \mathrm{MHz}, \mathrm{CDCl}_{3}\right): \delta=14.1,22.7,29.36,29.38,29.65,29.69$, 
29.7, 30.5, 30.6, 31.9, 35.3, 35.7, 38.7, 38.9, 51.8, 53.4, 116.6, 118.3, 119.2, $119.6,126.2,127.9,128.1,130.6,130.7,133.5,134.9,144.8,144.9$, $167.5 \mathrm{ppm}$; IR $(\mathrm{KBr}): \tilde{v}=3317,3123,3028,2952,2923,2852,1723,1635$, 1480, 1466, 1434, 1321, 1312, 1203, 1168, 983, 917, 790, $732 \mathrm{~cm}^{-1}$; HRMS (FAB): $m / z$ calcd for $\mathrm{C}_{63} \mathrm{H}_{89} \mathrm{~N}_{4} \mathrm{O}_{2}: 933.6986\left[M^{+}+\mathrm{H}\right]$; found: 933.6978 .

\section{H,23H-5-(4-Nitrophenyl)-10,15,20-tris(4-octyloxyphenyl)-porphyrin} (5d): ${ }^{[12]}$ TFA $\left(0.75 \mathrm{~mL}, 5 \times 10^{-3} \mathrm{M}\right)$ was added to a mixture of $\mathbf{3 d}(0.76 \mathrm{~g}$, $5 \mathrm{mmol}), 4 \mathbf{b}^{[23]}(3.52 \mathrm{~g}, 15 \mathrm{mmol})$, and pyrrole $(1.34 \mathrm{~g}, 20 \mathrm{mmol})$ in $\mathrm{CH}_{2} \mathrm{Cl}_{2}(2 \mathrm{~L})$ and stirred for $2.5 \mathrm{~h}$, then quenched with DDQ $(3.40 \mathrm{~g}$, $15 \mathrm{mmol}$ ) for $1 \mathrm{~h}$ at RT. The solution was concentrated and filtered through an alumina pad to remove the quinone and polypyrrylmethine species. The solvent was removed in vacuo and the residue was purified by column chromatography on silica gel (eluent hexane/ $\mathrm{CH}_{2} \mathrm{Cl}_{2} 7: 3$ ) to give 5 d $(0.42 \mathrm{~g}, 8 \%)$. M.p. $202-203^{\circ} \mathrm{C} ;{ }^{1} \mathrm{H} \mathrm{NMR}\left(300 \mathrm{MHz}, \mathrm{CDCl}_{3}\right): \delta=$ $-2.74(\mathrm{~s}, 2 \mathrm{H} ; \mathrm{NH}), 0.94\left(\mathrm{t},{ }^{3} \mathrm{~J}(\mathrm{H}, \mathrm{H})=6.3 \mathrm{~Hz}, 9 \mathrm{H} ; \mathrm{CH}_{3}\right), 1.35-1.64(\mathrm{~m}$, $30 \mathrm{H} ; \mathrm{CH}_{2}$ ), 1.95 (quint, $\left.{ }^{3} J(\mathrm{H}, \mathrm{H})=6.3 \mathrm{~Hz}, 6 \mathrm{H} ; \mathrm{CH}_{2}\right), 4.18\left(\mathrm{t},{ }^{3} J(\mathrm{H}, \mathrm{H})=\right.$ $\left.5.9 \mathrm{~Hz}, 6 \mathrm{H} ; \mathrm{OCH}_{2}\right), 7.22\left(\mathrm{~d},{ }^{3} J(\mathrm{H}, \mathrm{H})=8.4 \mathrm{~Hz}, 6 \mathrm{H} ; \mathrm{ArH}\right), 8.06\left(\mathrm{dd},{ }^{3} J-\right.$ $(\mathrm{H}, \mathrm{H})=8.4,3.3 \mathrm{~Hz}, 6 \mathrm{H} ; \mathrm{ArH}), 8.31\left(\mathrm{~d},{ }^{3} J(\mathrm{H}, \mathrm{H})=8.4 \mathrm{~Hz}, 2 \mathrm{H} ; \mathrm{ArH}\right), 8.55$ $(\mathrm{d}, J=8.4 \mathrm{~Hz}, 2 \mathrm{H} ; \mathrm{ArH}), 8.67(\mathrm{~d}, J=4.8 \mathrm{~Hz}, 2 \mathrm{H} ; \beta-\mathrm{H}), 8.87(\mathrm{~s}, 4 \mathrm{H} ; \beta-\mathrm{H})$, $8.89 \mathrm{ppm}\left(\mathrm{d},{ }^{3} \mathrm{~J}(\mathrm{H}, \mathrm{H})=4.8 \mathrm{~Hz}, 2 \mathrm{H} ; \beta-\mathrm{H}\right) ;{ }^{13} \mathrm{C} \mathrm{NMR}\left(75 \mathrm{MHz}, \mathrm{CDCl}_{3}\right)$ : $\delta=14.1,22.7,26.3,29.3,29.5,31.9,68.4,112.9,114.8,116.2,120.5,121.0$, 121.8, 129.8, 131.4, 132.0, 134.1, 134.2, 135.1, 135.6, 146.9, 147.7, 149.4, $159.2 \mathrm{ppm}$; IR $(\mathrm{KBr}): \tilde{v}=2925,2855,1606,1510,1469,1347,1284,1245$, 1174, 1108, 966, 847, 801, $735 \mathrm{~cm}^{-1}$; LRMS (MALDI): $\mathrm{m} / \mathrm{z}(\%): 1044.0$ (100) $\left[M^{+}\right], 1045.0(80)\left[M^{+}+\mathrm{H}\right]$; elemental analysis calcd $(\%)$ for $\mathrm{C}_{68} \mathrm{H}_{77} \mathrm{~N}_{5} \mathrm{O}_{5}$ : C 78.20, $\mathrm{H}$ 7.43, N 6.71; found: C 78.34, H 7.84, N 5.90.

\section{H,23H-5-(4-Hydroxyphenyl)-10,15,20-tris(4-octyloxyphenyl)-porphyrin} (5e) $;^{[24]}$ TFA $\left(1.5 \mathrm{~mL}, 10^{-2} \mathrm{M}\right)$ was added slowly to a mixture of $\mathbf{4 b}$ $(7.02 \mathrm{~g}, 30 \mathrm{mmol}), 3 \mathrm{a}(1.22 \mathrm{~g}, 10 \mathrm{mmol})$, and pyrrole $(2.68 \mathrm{~g}, 40 \mathrm{mmol})$ in $\mathrm{CH}_{2} \mathrm{Cl}_{2}(2 \mathrm{~L})$ and the mixture was stirred for $2.5 \mathrm{~h}$, then quenched by addition of $p$-chloranil $(7.44 \mathrm{~g}, 30 \mathrm{mmol})$. After $3 \mathrm{~h}$ at RT, $\mathrm{NaHCO}_{3}(10 \mathrm{~g})$ was added to neutralize TFA. The crude mixture was concentrated and filtered through an alumina pad to remove quinone and polypyrrylmethene species. The solvent was removed in vacuo and the residue was purified by column chromatography on silica gel (eluent $\mathrm{CH}_{2} \mathrm{Cl}_{2}$ ) to give 5e as a purple solid $(1.217 \mathrm{~g}, 12 \%)$. M.p. $228-229^{\circ} \mathrm{C}$; ${ }^{1} \mathrm{H}$ NMR $\left(300 \mathrm{MHz}, \mathrm{CDCl}_{3}\right): \delta=-2.74(\mathrm{~s}, 2 \mathrm{H} ; \mathrm{NH}), 0.93\left(\mathrm{t},{ }^{3} J(\mathrm{H}, \mathrm{H})=6.6 \mathrm{~Hz}, 9 \mathrm{H}\right.$; $\left.\mathrm{CH}_{3}\right), 1.35-1.52\left(\mathrm{~m}, 24 \mathrm{H} ; \mathrm{CH}_{2}\right), 1.60$ (quint., ${ }^{3} \mathrm{~J}(\mathrm{H}, \mathrm{H})=6.6 \mathrm{~Hz}, 6 \mathrm{H}$; $\mathrm{CH}_{2}$ ), 1.95 (quint., $\left.{ }^{3} \mathrm{~J}(\mathrm{H}, \mathrm{H})=6.6 \mathrm{~Hz}, 6 \mathrm{H} ; \mathrm{CH}_{2}\right), 4.19\left(\mathrm{t},{ }^{3} \mathrm{~J}(\mathrm{H}, \mathrm{H})=6.6 \mathrm{~Hz}\right.$, $\left.4 \mathrm{H} ; \mathrm{OCH}_{2}\right), 4.20\left(\mathrm{t},{ }^{3} \mathrm{~J}(\mathrm{H}, \mathrm{H})=6.6 \mathrm{~Hz}, 2 \mathrm{H} ; \mathrm{OCH}_{2}\right), 5.01(\mathrm{~s}, 1 \mathrm{H} ; \mathrm{OH})$, $7.02\left(\mathrm{~d},{ }^{3} J(\mathrm{H}, \mathrm{H})=8.4 \mathrm{~Hz}, 2 \mathrm{H}\right), 7.22\left(\mathrm{~d},{ }^{3} J(\mathrm{H}, \mathrm{H})=8.4 \mathrm{~Hz}, 4 \mathrm{H}\right), 7.24\left(\mathrm{~d},{ }^{3} \mathrm{~J}-\right.$ $(\mathrm{H}, \mathrm{H})=8.4 \mathrm{~Hz}, 2 \mathrm{H}), 8.00\left(\mathrm{~d},{ }^{3} J(\mathrm{H}, \mathrm{H})=8.4 \mathrm{~Hz}, 2 \mathrm{H}\right), 8.07\left(\mathrm{~d},{ }^{3} J(\mathrm{H}, \mathrm{H})=\right.$ $8.4 \mathrm{~Hz}, 4 \mathrm{H}), 8.09\left(\mathrm{~d},{ }^{3} J(\mathrm{H}, \mathrm{H})=8.4 \mathrm{~Hz}, 2 \mathrm{H}\right), 8.82\left(\mathrm{~d},{ }^{3} J(\mathrm{H}, \mathrm{H})=5.1 \mathrm{~Hz}\right.$, $2 \mathrm{H} ; \beta-\mathrm{H}), 8.85\left(\mathrm{~d},{ }^{3} J(\mathrm{H}, \mathrm{H})=5.1 \mathrm{~Hz}, 2 \mathrm{H} ; \beta-\mathrm{H}\right), 8.86 \mathrm{ppm}(\mathrm{s}, 4 \mathrm{H} ; \beta-\mathrm{H})$; ${ }^{13} \mathrm{C} \mathrm{NMR}\left(75 \mathrm{MHz}, \mathrm{CDCl}_{3}\right): \delta=14.4,22.9,26.4,29.5,29.7,32.1,68.3$, $112.5,113.42,113.43,119.3,119.68,119.72,130.8,134.15,134.18,134.5$, $135.33,135.38,135.41,155.0,158.61,158.62 \mathrm{ppm} ; \mathrm{IR}(\mathrm{KBr}) \tilde{v}=3357$, 2924, 2852, 1605, 1571, 1509, 1467, 1349, 1283, 1244, 1174, 1108, 966, 840, 803, $738 \mathrm{~cm}^{-1}$; LRMS (MALDI): $\mathrm{m} / z$ (\%): $1015.6(100)\left[M^{+}+\mathrm{H}\right], 1016.6$ (72) $\left[M^{+}+2\right]$; HRMS (MALDI): $m / z$ calcd for $\mathrm{C}_{68} \mathrm{H}_{79} \mathrm{~N}_{4} \mathrm{O}_{4}: 1015.6053$ $\left[M^{+}+\mathrm{H}\right]$; found: 1015.6096 .

\section{H,23H-5-(4-Aminophenyl)-10,15,20-tris(4-octoxyphenyl)-porphyrin} (5f) ${ }^{[25]}$ A mixture of $5 d(522 \mathrm{mg}, 0.5 \mathrm{mmol}), \mathrm{SnCl}_{2} \cdot 2 \mathrm{H}_{2} \mathrm{O} \quad(564 \mathrm{mg}$, $2.5 \mathrm{mmol})$, and $\mathrm{HCl}(35 \%, 3 \mathrm{~mL})$ in $\mathrm{THF}(25 \mathrm{~mL})$ was stirred at $\mathrm{RT}$ under argon for $24 \mathrm{~h}$. The solvent was removed in vacuo and the residue was dissolved in $\mathrm{CH}_{2} \mathrm{Cl}_{2}(50 \mathrm{~mL})$. Sodium hydroxide $(10 \%)$ was added until the $\mathrm{pH}$ value was basic, then the organic layer was separated and dried $\left(\mathrm{MgSO}_{4}\right)$ and the solvent was removed in vacuo to give a residue that was purified by column chromatography on silica gel (eluent $\left.\mathrm{CH}_{2} \mathrm{Cl}_{2}\right)$ to give $5 \mathbf{f}$ as a purple solid $(0.38 \mathrm{~g}, 75 \%)$. M.p. $203-206^{\circ} \mathrm{C}$; ${ }^{1} \mathrm{H}$ NMR $\left(300 \mathrm{MHz}, \mathrm{CDCl}_{3}\right): \delta=-2.73(\mathrm{~s}, 2 \mathrm{H} ; \mathrm{NH}), 0.93\left(\mathrm{t},{ }^{3} J(\mathrm{H}, \mathrm{H})=\right.$ $\left.6.6 \mathrm{~Hz}, 9 \mathrm{H} ; \mathrm{CH}_{3}\right), 1.35-1.49\left(\mathrm{~m}, 24 \mathrm{H} ; \mathrm{CH}_{2}\right), 1.60$ (quint., ${ }^{3} \mathrm{~J}(\mathrm{H}, \mathrm{H})=$ $6.6 \mathrm{~Hz}, 6 \mathrm{H} ; \mathrm{CH}_{2}$ ), 1.95 (quint., ${ }^{3} J(\mathrm{H}, \mathrm{H})=6.6 \mathrm{~Hz}, 6 \mathrm{H} ; \mathrm{CH}_{2}$ ), 3.93 (brs, $\left.2 \mathrm{H} ; \mathrm{NH}_{2}\right), 4.19\left(\mathrm{t},{ }^{3} J(\mathrm{H}, \mathrm{H})=6.6 \mathrm{~Hz}, 6 \mathrm{H} ; \mathrm{OCH}_{2}\right), 6.99\left(\mathrm{~d},{ }^{3} J(\mathrm{H}, \mathrm{H})=\right.$ $7.8 \mathrm{~Hz}, 2 \mathrm{H} ; \mathrm{ArH}), 7.23\left(\mathrm{~d},{ }^{3} J(\mathrm{H}, \mathrm{H})=8.4 \mathrm{~Hz}, 6 \mathrm{H} ; \mathrm{ArH}\right), 7.96\left(\mathrm{~d},{ }^{3} J-\right.$ $(\mathrm{H}, \mathrm{H})=7.8 \mathrm{~Hz}, 2 \mathrm{H} ; \mathrm{ArH}), 8.09\left(\mathrm{~d},{ }^{3} \mathrm{~J}(\mathrm{H}, \mathrm{H})=8.4 \mathrm{~Hz}, 6 \mathrm{H} ; \mathrm{ArH}\right), 8.85(\mathrm{~d}$, $\left.{ }^{3} J(\mathrm{H}, \mathrm{H})=4.8 \mathrm{~Hz}, 2 \mathrm{H} ; \beta-\mathrm{H}\right), 8.86(\mathrm{~s}, 4 \mathrm{H} ; \beta-\mathrm{H}), 8.91 \mathrm{ppm}\left(\mathrm{d},{ }^{3} J(\mathrm{H}, \mathrm{H})=\right.$ $4.8 \mathrm{~Hz}, 2 \mathrm{H} ; \beta-\mathrm{H}) ;{ }^{13} \mathrm{C}$ NMR $\left(75 \mathrm{MHz}, \mathrm{CDCl}_{3}\right): \delta=14.2,22.7,26.3,29.4$,
$29.5,31.9,68.3,112.7,113.4,119.7,119.8,120.5,130.7,131.0,132.6,134.5$, 135.6, 135.7, 146.0, 159.0 ppm; IR (KBr): $\tilde{v}=3390,3318,2923,2852,1606$, $1561,1509,1466,1243,1175,966,805,741 \mathrm{~cm}^{-1}$; LRMS (MALDI): $\mathrm{m} / z$ $(\%): 1016.5$ (100) $\left[M^{+}+3 \mathrm{H}\right]$; HRMS (MALDI): $\mathrm{m} / \mathrm{z}$ calcd for $\mathrm{C}_{68} \mathrm{H}_{80} \mathrm{~N}_{5} \mathrm{O}_{3}: 1014.6232\left[M^{+}+\mathrm{H}\right]$; found: 1014.6256; elemental analysis calcd (\%) for $\mathrm{C}_{68} \mathrm{H}_{79} \mathrm{~N}_{5} \mathrm{O}_{3}$ : C 80.51, H 7.85, N 6.90; found: C 80.29, H $8.26, \mathrm{~N} 6.35$.

5-\{4-[2-(Methoxycarbonyl)-1-vinyl]phenyl\}-10,15,20-tris-(undecyl)porphyrinzinc(II) (6a): Zinc acetate $(730 \mathrm{mg}, 3.32 \mathrm{mmol})$ was added to a solution of $\mathbf{5 c}(620 \mathrm{mg}, 0.66 \mathrm{mmol})$ in methanol $(10 \mathrm{~mL})$ and $\mathrm{CH}_{2} \mathrm{Cl}_{2}$ $(30 \mathrm{~mL})$. The mixture was stirred at RT in the dark for $3 \mathrm{~h}$ and then washed with $\mathrm{NaHCO}_{3}(5 \%)$ and brine and then dried $\left(\mathrm{MgSO}_{4}\right)$. The solvent was removed in vacuo to give a residue that was purified by column chromatography on silica gel (eluent hexane $/ \mathrm{CH}_{2} \mathrm{Cl}_{2} / \mathrm{NEt}_{3} \quad 1: 1: 0.05$ ) to afford 6a $(580 \mathrm{mg}, 88 \%)$. M.p. $89-90{ }^{\circ} \mathrm{C} ;{ }^{1} \mathrm{H}$ NMR $\left(400 \mathrm{MHz}, \mathrm{CDCl}_{3}\right)$ : $\delta=0.86-0.91(\mathrm{~m}, 9 \mathrm{H}), 1.25-1.49(\mathrm{~m}, 36 \mathrm{H}), 1.49-1.60(\mathrm{~m}, 6 \mathrm{H}), 1.72-1.90$ $(\mathrm{m}, 6 \mathrm{H}), 2.33-2.48(\mathrm{~m}, 6 \mathrm{H}), 3.93(\mathrm{~s}, 3 \mathrm{H}), 4.58\left(\mathrm{t},{ }^{3} J(\mathrm{H}, \mathrm{H})=7.8 \mathrm{~Hz}, 2 \mathrm{H}\right)$, $4.72\left(\mathrm{t},{ }^{3} J(\mathrm{H}, \mathrm{H})=7.8 \mathrm{~Hz}, 4 \mathrm{H}\right), 6.76\left(\mathrm{~d},{ }^{3} J(\mathrm{H}, \mathrm{H})=16.1 \mathrm{~Hz}, 1 \mathrm{H}\right), 7.93(\mathrm{~d}$, $\left.{ }^{3} J(\mathrm{H}, \mathrm{H})=8.0 \mathrm{~Hz}, 2 \mathrm{H}\right), 8.06\left(\mathrm{~d},{ }^{3} J(\mathrm{H}, \mathrm{H})=16.1 \mathrm{~Hz}, 1 \mathrm{H}\right), 8.21 \quad\left(\mathrm{~d},{ }^{3} J-\right.$ $(\mathrm{H}, \mathrm{H})=8.0 \mathrm{~Hz}, 2 \mathrm{H}), 8.84\left(\mathrm{~d},{ }^{3} J(\mathrm{H}, \mathrm{H})=4.7 \mathrm{~Hz}, 2 \mathrm{H}\right), 9.19\left(\mathrm{~d},{ }^{3} J(\mathrm{H}, \mathrm{H})=\right.$ $4.7 \mathrm{~Hz}, 2 \mathrm{H}), 9.22\left(\mathrm{~d},{ }^{3} J(\mathrm{H}, \mathrm{H})=4.7 \mathrm{~Hz}, 2 \mathrm{H}\right), 9.37 \mathrm{ppm}\left(\mathrm{d},{ }^{3} J(\mathrm{H}, \mathrm{H})=\right.$ $4.7 \mathrm{~Hz}, 2 \mathrm{H}) ;{ }^{13} \mathrm{C} \mathrm{NMR}\left(100 \mathrm{MHz}, \mathrm{CDCl}_{3}\right): \delta=14.3,22.8,29.529 .80$, $29.85,29.88,30.9,32.0,35.3,35.5,38.9,39.0,51.9,117.3,117.9,119.7$, $126.1,128.0,128.2,128.4,131.1,133.1,134.9,144.8,145.4,148.1,148.5$, $148.8,149.1,167.4$ ppm; IR (KBr): $\tilde{v}=3123,3060,3025,2952,2923,2852$, 1723, 1633, 1603, 1466, 1435, 1322, 1205, 1167, 1078, 1007, 986, 932, 787, $712 \mathrm{~cm}^{-1}$; HRMS (FAB): $\mathrm{m} / z$ calcd for $\mathrm{C}_{63} \mathrm{H}_{86} \mathrm{~N}_{4} \mathrm{O}_{2} \mathrm{Zn}$ : $994.6042\left[M^{+}\right]$; found: 994.6044

\section{5-[4-(3-Hydroxy-1-propyl)phenyl]-10,15,20-tris-(undecyl)-porphyrin-} zinc(II) (6b): A mixture of $\mathbf{6 a}(500 \mathrm{mg}, 0.50 \mathrm{mmol})$ and $\mathrm{Pd} / \mathrm{C}(10 \mathrm{wt} \%$, $50 \mathrm{mg}, 0.05 \mathrm{mmol})$ in EtOH $(10 \mathrm{~mL})$ and THF $(20 \mathrm{~mL})$ was purged three times with hydrogen. The reaction system was then charged with hydrogen to $1 \mathrm{~atm}$ and stirred at RT for $24 \mathrm{~h}$. After filtration through Celite, the filtrate was evaporated in vacuo to give the corresponding hydrogenated product (410 mg, $83 \%$ ). M.p. $46-47^{\circ} \mathrm{C} ;{ }^{1} \mathrm{H}$ NMR (400 $\mathrm{MHz}, \mathrm{CDCl}_{3}$ ): $\delta=0.86-0.91(\mathrm{~m}, 9 \mathrm{H}), 1.24-1.45(\mathrm{~m}, 36 \mathrm{H}), 1.75-1.86(\mathrm{~m}, 6 \mathrm{H}), 1.75-1.86$ $(\mathrm{m}, 6 \mathrm{H}), 2.35-2.49(\mathrm{~m}, 6 \mathrm{H}), 2.98\left(\mathrm{t},{ }^{3} \mathrm{~J}(\mathrm{H}, \mathrm{H})=7.8 \mathrm{~Hz}, 2 \mathrm{H}\right), 3.34\left(\mathrm{t},{ }^{3} J-\right.$ $(\mathrm{H}, \mathrm{H})=7.8 \mathrm{~Hz}, 2 \mathrm{H}), 3.84(\mathrm{~s}, 3 \mathrm{H}), 4.65\left(\mathrm{t},{ }^{3} J(\mathrm{H}, \mathrm{H})=7.6 \mathrm{~Hz}, 2 \mathrm{H}\right), 4.79(\mathrm{t}$, $\left.{ }^{3} J(\mathrm{H}, \mathrm{H})=7.8 \mathrm{~Hz}, 2 \mathrm{H}\right), 7.59\left(\mathrm{~d},{ }^{3} J(\mathrm{H}, \mathrm{H})=8.0 \mathrm{~Hz}, 2 \mathrm{H}\right), 8.11\left(\mathrm{~d},{ }^{3} J(\mathrm{H}, \mathrm{H})=\right.$ $8.0 \mathrm{~Hz}, 2 \mathrm{H}), 8.88\left(\mathrm{~d},{ }^{3} J(\mathrm{H}, \mathrm{H})=4.7 \mathrm{~Hz}, 2 \mathrm{H}\right), 9.26\left(\mathrm{~d},{ }^{3} J(\mathrm{H}, \mathrm{H})=4.7 \mathrm{~Hz}\right.$, $2 \mathrm{H}), 9.29\left(\mathrm{~d},{ }^{3} J(\mathrm{H}, \mathrm{H})=4.7 \mathrm{~Hz}, 2 \mathrm{H}\right), 9.41 \mathrm{ppm}\left(\mathrm{d},{ }^{3} J(\mathrm{H}, \mathrm{H})=4.7 \mathrm{~Hz}, 2 \mathrm{H}\right)$; ${ }^{13} \mathrm{C}$ NMR $\left(100 \mathrm{MHz}, \mathrm{CDCl}_{3}\right): \delta=14.1,22.7,29.4,29.7,29.8,30.68,30.74$, $31.0,31.9,35.2,35.4,36.0,38.7,38.8,51.8,118.5,119.6,126.3,128.0,128.2$, 128.3, 131.6, 134.6, 139.4, 141.3, 148.2, 148.9, 149.3, 149.4, $173.6 \mathrm{ppm}$; IR (KBr) $\tilde{v}=3123,3018,2958,2923,2852,1743,1526,1502,1466,1437$, 1344, 1210, 1077, 1008, 788, $712 \mathrm{~cm}^{-1}$; HRMS (FAB): $\mathrm{m} / z$ : calcd for $\mathrm{C}_{63} \mathrm{H}_{88} \mathrm{~N}_{4} \mathrm{O}_{2} \mathrm{Zn}$ : $996.6199\left[M^{+}\right]$; found: 996.6192.

DIBAL-H $(4.0 \mathrm{~mL}, 1.0 \mathrm{M}$ in THF, $4.0 \mathrm{mmol})$ was added slowly to a solution of hydrogenated product $(800 \mathrm{mg}, 0.8 \mathrm{mmol})$ in THF $(20 \mathrm{~mL})$ at $0{ }^{\circ} \mathrm{C}$ and stirred under a $\mathrm{N}_{2}$ atmosphere. The reaction mixture was allowed to stir for $2 \mathrm{~h}$ at RT, then quenched by slowly pouring into saturated $\mathrm{NH}_{4} \mathrm{Cl}$. After the remaining DIBAL-H was completely quenched, the gel-like organic layer was acidified with $6 \mathrm{M} \mathrm{HCl}$ and extracted with $\mathrm{CH}_{2} \mathrm{Cl}_{2}$. The combined organic layers were washed with $\mathrm{NaOH}(10 \%)$ and brine and then dried $\left(\mathrm{MgSO}_{4}\right)$ and evaporated in vacuo to afford $6 \mathbf{b}(730 \mathrm{mg}$, $94 \%$ ). M.p. $50-51{ }^{\circ} \mathrm{C} ;{ }^{1} \mathrm{H}$ NMR $\left(400 \mathrm{MHz}, \mathrm{CDCl}_{3}\right): \delta=0.86-0.91(\mathrm{~m}$, $9 \mathrm{H}), 1.13\left(\mathrm{t},{ }^{3} \mathrm{~J}(\mathrm{H}, \mathrm{H})=5.3 \mathrm{~Hz}, 1 \mathrm{H}\right), 1.27-1.48(\mathrm{~m}, 36 \mathrm{H}), 1.49-1.60(\mathrm{~m}$, $6 \mathrm{H}), 1.76-1.86(\mathrm{~m}, 6 \mathrm{H}), 1.98-2.13(\mathrm{~m}, 2 \mathrm{H}), 2.38-2.51(\mathrm{~m}, 6 \mathrm{H}), 2.95\left(\mathrm{t},{ }^{3} J-\right.$ $(\mathrm{H}, \mathrm{H})=7.7 \mathrm{~Hz}, 2 \mathrm{H}), 3.61-3.67(\mathrm{~m}, 2 \mathrm{H}), 4.65\left(\mathrm{t},{ }^{3} J(\mathrm{H}, \mathrm{H})=8.1 \mathrm{~Hz}, 2 \mathrm{H}\right)$, $4.77\left(\mathrm{t},{ }^{3} J(\mathrm{H}, \mathrm{H})=8.1 \mathrm{~Hz}, 4 \mathrm{H}\right), 7.51\left(\mathrm{~d},{ }^{3} J(\mathrm{H}, \mathrm{H})=7.9 \mathrm{~Hz}, 2 \mathrm{H}\right), 8.09\left(\mathrm{~d},{ }^{3} J\right.$ $(\mathrm{H}, \mathrm{H})=7.9 \mathrm{~Hz}, 2 \mathrm{H}), 8.88\left(\mathrm{~d},{ }^{3} J(\mathrm{H}, \mathrm{H})=4.7 \mathrm{~Hz}, 2 \mathrm{H}\right), 9.25\left(\mathrm{~d},{ }^{3} J(\mathrm{H}, \mathrm{H})=\right.$ $4.7 \mathrm{~Hz}, 2 \mathrm{H}), 9.27\left(\mathrm{~d},{ }^{3} J(\mathrm{H}, \mathrm{H})=4.7 \mathrm{~Hz}, 2 \mathrm{H}\right), 9.39 \mathrm{ppm}\left(\mathrm{d},{ }^{3} J(\mathrm{H}, \mathrm{H})=\right.$ $4.7 \mathrm{~Hz}, 2 \mathrm{H}) ;{ }^{13} \mathrm{C}$ NMR $\left(100 \mathrm{MHz}, \mathrm{CDCl}_{3}\right): \delta=14.1,22.7,29.4,29.7,29.8$ $30.8,31.8,31.9,33.9,35.37,35.44,38.8,38.9,62.1,118.7,119.55,119.62$, $126.4,128.1,128.28,128.33,131.7,134.5,140.5,140.8,148.4,149.0,149.3$ 149.4 ppm; IR (KBr): $\tilde{v}=3326,3123,3018,2955,2923,2852,1608,1557$ $1525,1502,1466,1343,1298,1209,1076,1056,1009,788,712 \mathrm{~cm}^{-1}$; 
HRMS (FAB): $m / z$ calcd for $\mathrm{C}_{62} \mathrm{H}_{88} \mathrm{~N}_{4} \mathrm{OZn}$ : $968.6250\left[M^{+}\right]$; found: 968.6257.

\section{4-\{4-[(tert-Butoxycarbonyl)methoxycarbonyl]phenyl\}-4-azatri-cy-}

clo[5.2.1.0 $\left.{ }^{2,6}\right]$ dec-8-ene (9a): tert-Butyl bromoacetate $(0.97 \mathrm{~g}, 5.0 \mathrm{mmol})$ was added to a mixture of $7(1.02 \mathrm{~g}, 4.0 \mathrm{mmol})$ and $\mathrm{Cs}_{2} \mathrm{CO}_{3}(0.65 \mathrm{~g}$, $2.0 \mathrm{mmol})$ in DMF $(25 \mathrm{~mL})$, then the mixture was heated to $50^{\circ} \mathrm{C}$ for 30 min before being cooled to RT. $\mathrm{CH}_{2} \mathrm{Cl}_{2}(70 \mathrm{~mL})$ was added and the mixture was poured into saturated $\mathrm{NaHCO}_{3}$. The organic layer was separated and washed with aqueous $\mathrm{NaHCO}_{3}(3 \times 50 \mathrm{~mL})$ and water $(2 \times$ $50 \mathrm{~mL})$ and then dried $\left(\mathrm{MgSO}_{4}\right)$, and the solvent was removed in vacuo to give a residue that was flash purified by using column chromatography (eluent hexane/EtOAc 2:1) to give 9a $(1.40 \mathrm{~g}, 95 \%)$. M.p. $160-162^{\circ} \mathrm{C}$; ${ }^{1} \mathrm{H}$ NMR $\left(300 \mathrm{MHz}, \mathrm{CDCl}_{3}\right): \delta=1.48\left(\mathrm{~s}, 9 \mathrm{H} ; \mathrm{CH}_{3}\right), 1.52\left(\mathrm{~d},{ }^{2} J(\mathrm{H}, \mathrm{H})=\right.$ $8.1 \mathrm{~Hz}, 1 \mathrm{H}$; anti-10-H), $1.62\left(\mathrm{~d},{ }^{2} \mathrm{~J}(\mathrm{H}, \mathrm{H})=8.1 \mathrm{~Hz}, 1 \mathrm{H}\right.$; syn-10-H), 2.94 $2.95(\mathrm{~m}, 2 \mathrm{H}), 2.8(\mathrm{~s}, 2 \mathrm{H}), 3.08-3.10(\mathrm{~m}, 2 \mathrm{H}), 3.27-3.33(\mathrm{~m}, 2 \mathrm{H}), 4.67(\mathrm{~s}$, $\left.2 \mathrm{H}, \mathrm{CH}_{2} \mathrm{O}\right), 6.17(\mathrm{~s}, 2 \mathrm{H}$; vinyl- $\mathrm{H}), 6.39\left(\mathrm{~d},{ }^{3} \mathrm{~J}(\mathrm{H}, \mathrm{H})=9.0 \mathrm{~Hz}, 2 \mathrm{H}\right)$, $7.91 \mathrm{ppm}\left(\mathrm{d},{ }^{3} J(\mathrm{H}, \mathrm{H})=9.0 \mathrm{~Hz}, 2 \mathrm{H}\right) ;{ }^{13} \mathrm{C} \mathrm{NMR}\left(100 \mathrm{MHz}, \mathrm{CDCl}_{3}\right): \delta=$ 28.0, 45.4, 46.6, 50.4, 52.1, 61.1, 82.0, 110.9, 115.3, 131.6, 135.8, 150.6, 166.3, $167.6 \mathrm{ppm}$; IR (KBr): $\tilde{v}=2978,2942,2867,1745,1696,1609,1526$, 1482, 1426, 1386, 1368, 1298, 1230, 1166, 1106, $771 \mathrm{~cm}^{-1}$; LRMS (ESI): $m / z(\%): 370.2(60)\left[M^{+}+\mathrm{H}\right]$; HRMS (ESI): $\mathrm{m} / z$ calcd for $\mathrm{C}_{22} \mathrm{H}_{27} \mathrm{NO}_{4}$ : $370.2026\left[M^{+}+\mathrm{H}\right]$; found: 370.2013 .

\section{4-\{4-[(Hydroxycarbonyl)methoxycarbonyl]phenyl\}-4-azatri-cyclo}

[5.2.1.0 ${ }^{2,6}$ ]dec-8-ene (9b): A solution of $9 \mathrm{a}(1.11 \mathrm{~g}, 3.0 \mathrm{mmol})$ and $\mathrm{CF}_{3} \mathrm{CO}_{2} \mathrm{H}(50 \%)$ in $\mathrm{CH}_{2} \mathrm{Cl}_{2}(30 \mathrm{~mL})$ was stirred for $3 \mathrm{~h}$ and then evaporated in vacuo to afford an oily residue. A small amount of water was added and the mixture was neutralized with saturated $\mathrm{NaHCO}_{3}$. The white precipitate was collected and washed with water and hexane and then dried under vacuum to give 9b $(1.0 \mathrm{~g}, 91 \%)$. M.p. $200-201^{\circ} \mathrm{C}$; ${ }^{1} \mathrm{H}$ NMR $\left(300 \mathrm{MHz}, \mathrm{CDCl}_{3}\right): \delta=1.49$ (apparent s, $2 \mathrm{H}$; bridging $\mathrm{CH}_{2}$ ), 2.90-2.94 (m, 4H), 3.06-3.8 (m, 2H), 3.25-3.29 (m, 2H), 4.70 (s, 2H; $\left.\mathrm{CH}_{2} \mathrm{O}\right), 6.17\left(\mathrm{~s}, 2 \mathrm{H}\right.$; vinyl-H), $6.45\left(\mathrm{~d},{ }^{3} J(\mathrm{H}, \mathrm{H})=9.0 \mathrm{~Hz}, 2 \mathrm{H}\right), 7.75\left(\mathrm{~d},{ }^{3} J-\right.$ $(\mathrm{H}, \mathrm{H})=9.0 \mathrm{~Hz}, 2 \mathrm{H}), 12.99 \mathrm{ppm}(\mathrm{brs}, 1 \mathrm{H} ; \mathrm{COOH}) ;{ }^{13} \mathrm{C} \mathrm{NMR}(100 \mathrm{MHz}$, $\left.\mathrm{CDCl}_{3}\right): \delta=44.7,46.0,50.0,51.5,60.3,111.0,114.4,130.9,135.7,150.3$, 165.4, $169.5 \mathrm{ppm}$; IR (KBr): $\tilde{v}=3060,2964,2941,2886,2848,1735,1714$, 1608, 1526, 1477, 1433, 1411, 1379, 1278, 1244, 1188, 1122, $767 \mathrm{~cm}^{-1}$; LRMS (ESI): $m / z(\%): 312.2\left(\left[M^{+}-\mathrm{H}\right]\right.$ (100); HRMS (ESI): $\mathrm{m} / z$ calcd for $\mathrm{C}_{18} \mathrm{H}_{20} \mathrm{NO}_{4}$ : $314.1397\left[M^{+}+\mathrm{H}\right]$; found: 314.1387 ; elemental analysis calcd (\%) for $\mathrm{C}_{18} \mathrm{H}_{19} \mathrm{NO}_{4}$ : C 68.99, $\mathrm{H} 6.11, \mathrm{~N}$ 4.47; found: $\mathrm{C} 68.44, \mathrm{H}$ $6.12, \mathrm{~N} 4.28$

Compound 11 a: Oxalyl chloride $(0.31 \mathrm{~mL}, 3.7 \mathrm{mmol})$ and DMF (one drop) were added to a solution of $7(390 \mathrm{mg}, 1.5 \mathrm{mmol})$ in $\mathrm{CH}_{2} \mathrm{Cl}_{2}$ $(20 \mathrm{~mL})$ at $0^{\circ} \mathrm{C}$. The mixture was gradually warmed to RT and stirred for $1 \mathrm{~h}$, then the solvent was removed in vacuo to give $\mathbf{8}$, which was used for the next reaction without further purification.

Next, compound 8 in $\mathrm{CH}_{2} \mathrm{Cl}_{2}(20 \mathrm{~mL})$ was added to a mixture of $\mathbf{5} \mathbf{a}$ (880 mg, $1.0 \mathrm{mmol}), \mathrm{NEt}_{3}(2.0 \mathrm{~mL})$, and DMAP (trace amount) in $\mathrm{CH}_{2} \mathrm{Cl}_{2}(20 \mathrm{~mL})$ at $0^{\circ} \mathrm{C}$. The mixture was stirred at $\mathrm{RT}$ for $17 \mathrm{~h}$, then saturated $\mathrm{NaHCO}_{3}$ was added and the solution was washed with water and brine and then dried $\left(\mathrm{MgSO}_{4}\right)$. The solvent was removed in vacuo and the residue was purified by column chromatography on silica gel (eluent hexane $/ \mathrm{CH}_{2} \mathrm{Cl}_{2} / \mathrm{NEt}_{3}$ 1:1:0.05) to give 11 a $(1.03 \mathrm{~g}, 93 \%)$. M.p. $92-93{ }^{\circ} \mathrm{C}$; ${ }^{1} \mathrm{H}$ NMR $\left(400 \mathrm{MHz}, \mathrm{CDCl}_{3}\right): \delta=-2.64(\mathrm{~s}, 2 \mathrm{H} ; \mathrm{NH}), 0.87-0.93(\mathrm{~m}, 9 \mathrm{H}$; $\left.\mathrm{CH}_{3}\right), 1.25-1.47\left(\mathrm{~m}, 36 \mathrm{H} ; \mathrm{CH}_{2}\right), 1.47-1.62\left(\mathrm{~m}, 7 \mathrm{H} ; \mathrm{CH}_{2} ; \mathrm{CH}\right.$ on norbornene $), 1.65\left(\mathrm{~d},{ }^{2} \mathrm{~J}(\mathrm{H}, \mathrm{H})=8.2 \mathrm{~Hz}, 1 \mathrm{H} ; \mathrm{CH}\right.$ on norbornene $), 1.78-1.87(\mathrm{~m}$, $\left.6 \mathrm{H} ; \mathrm{CH}_{2}\right), 2.50-2.58\left(\mathrm{~m}, 6 \mathrm{H} ; \mathrm{CH}_{2}\right), 3.00-3.04(\mathrm{~m}, 4 \mathrm{H} ; \mathrm{H}$ on norbornene), 3.06-3.09 (m, 2H; $\mathrm{H}$ on norbornene), 3.29-3.34 (m, 2H; $\mathrm{H}$ on norbornene), 4.91-4.98 (m, 6H; $\left.\mathrm{CH}_{2}\right), 6.21(\mathrm{~s}, 2 \mathrm{H}$; alkenyl $\mathrm{H}$ on norbornene), $6.53\left(\mathrm{~d},{ }^{3} J(\mathrm{H}, \mathrm{H})=8.9 \mathrm{~Hz}, 2 \mathrm{H} ; \operatorname{ArH}\right), 7.61\left(\mathrm{~d},{ }^{3} J(\mathrm{H}, \mathrm{H})=8.4 \mathrm{~Hz}, 2 \mathrm{H}\right.$; $\operatorname{ArH}), 8.20\left(\mathrm{~d},{ }^{3} J(\mathrm{H}, \mathrm{H})=8.4 \mathrm{~Hz}, 2 \mathrm{H} ; \operatorname{ArH}\right), 8.21\left(\mathrm{~d},{ }^{3} J(\mathrm{H}, \mathrm{H})=8.9 \mathrm{~Hz}\right.$, $2 \mathrm{H} ; \mathrm{ArH}), 8.90\left(\mathrm{~d},{ }^{3} \mathrm{~J}(\mathrm{H}, \mathrm{H})=4.8 \mathrm{~Hz}, 2 \mathrm{H} ; \beta-\mathrm{H}\right), 9.40\left(\mathrm{~d},{ }^{3} \mathrm{~J}(\mathrm{H}, \mathrm{H})=4.8 \mathrm{~Hz}\right.$, $2 \mathrm{H} ; \beta-\mathrm{H}), 9.51\left(\mathrm{~d},{ }^{3} J(\mathrm{H}, \mathrm{H})=4.8 \mathrm{~Hz}, 2 \mathrm{H} ; \beta-\mathrm{H}\right), 9.54 \mathrm{ppm}\left(\mathrm{d},{ }^{3} J(\mathrm{H}, \mathrm{H})=\right.$ $4.8 \mathrm{~Hz}, 2 \mathrm{H} ; \beta-\mathrm{H}) ;{ }^{13} \mathrm{C}$ NMR $\left(100 \mathrm{MHz}, \mathrm{CDCl}_{3}\right): \delta=14.3,22.8,29.5$, $29.77,29.84,29.86,30.7,30.8,32.0,35.5,35.9,38.8,39.0,45.4,46.7,50.5$, $52.1,111.0,115.2,117.1,119.0,119.3,120.0127 .9,128.3,132.0,135.0$, $135.6,139.4,150.6,151.0,165.6 \mathrm{ppm} ; \mathrm{IR}(\mathrm{KBr}): \tilde{v}=3313,3126,3060$, 2955, 2923, 2852,1721,1603, 1527, 1474, 1380, 1272, 1205, 1175, 1164,
1058, 967, 791, $734 \mathrm{~cm}^{-1}$; HRMS (FAB): $\mathrm{m} / z$ calcd for $\mathrm{C}_{75} \mathrm{H}_{100} \mathrm{~N}_{5} \mathrm{O}_{2}$ : $1102.7877\left[M^{+}+\mathrm{H}\right]$; found: 1102.7886 .

Compound 11b: In a manner similar to that described above for $11 \mathrm{a}$ compound $\mathbf{5 g}^{[15]}$ was converted to $\mathbf{1 1 b}(0.37 \mathrm{~g}, 74 \%)$. M.p. $90-91^{\circ} \mathrm{C}$; ${ }^{1} \mathrm{H}$ NMR $\left(400 \mathrm{MHz}, \mathrm{CDCl}_{3}\right): \delta=-2.65(\mathrm{~s}, 2 \mathrm{H} ; \mathrm{NH}), 0.86-0.92(\mathrm{~m}, 9 \mathrm{H}$ $\left.\mathrm{CH}_{3}\right), 1.27-1.42\left(\mathrm{~m}, 36 \mathrm{H} ; \mathrm{CH}_{2}\right), 1.45-1.56\left(\mathrm{~m}, 7 \mathrm{H} ; \mathrm{CH}_{2} ; \mathrm{CH}\right.$ on norbornene $), 1.63\left(\mathrm{~d},{ }^{2} J(\mathrm{H}, \mathrm{H})=8.3 \mathrm{~Hz}, 1 \mathrm{H} ; \mathrm{CH}\right.$ on norbornene $), 1.77-1.85(\mathrm{~m}$, $\left.6 \mathrm{H} ; \mathrm{CH}_{2}\right), 2.50-2.56\left(\mathrm{~m}, 6 \mathrm{H} ; \mathrm{CH}_{2}\right), 2.97-3.01(\mathrm{~m}, 4 \mathrm{H} ; \mathrm{H}$ on norbornene), $3.07-3.10(\mathrm{~m}, 2 \mathrm{H} ; \mathrm{H}$ on norbornene $), 3.29-3.32(\mathrm{~m}, 2 \mathrm{H} ; \mathrm{H}$ on norbornene), 4.91-4.98 (m, 6H; $\left.\mathrm{CH}_{2}\right), 5.70\left(\mathrm{~s}, 2 \mathrm{H} ; \mathrm{OCH}_{2}\right), 6.19(\mathrm{~s}, 2 \mathrm{H}$; alkenyl $\mathrm{H}$ on norbornene), $6.47\left(\mathrm{~d},{ }^{3} J(\mathrm{H}, \mathrm{H})=9.0 \mathrm{~Hz}, 2 \mathrm{H} ; \mathrm{ArH}\right), 7.81\left(\mathrm{~d},{ }^{3} J\right.$ $(\mathrm{H}, \mathrm{H})=8.0 \mathrm{~Hz}, 2 \mathrm{H} ; \mathrm{ArH}), 8.10\left(\mathrm{~d},{ }^{3} \mathrm{~J}(\mathrm{H}, \mathrm{H})=9.0 \mathrm{~Hz}, 2 \mathrm{H} ; \mathrm{ArH}\right), 8.17(\mathrm{~d}$, $\left.{ }^{3} J(\mathrm{H}, \mathrm{H})=8.0 \mathrm{~Hz}, 2 \mathrm{H} ; \mathrm{ArH}\right), 8.83\left(\mathrm{~d},{ }^{3} J(\mathrm{H}, \mathrm{H})=4.8 \mathrm{~Hz}, 2 \mathrm{H} ; \beta-\mathrm{H}\right), 9.38(\mathrm{~d}$ $\left.{ }^{3} J(\mathrm{H}, \mathrm{H})=4.8 \mathrm{~Hz}, \quad 2 \mathrm{H} ; \quad \beta-\mathrm{H}\right), 9.50 \quad\left(\mathrm{~d},{ }^{3} J(\mathrm{H}, \mathrm{H})=4.9 \mathrm{~Hz}, \quad 2 \mathrm{H} ; \quad \beta-\mathrm{H}\right)$ $9.53 \mathrm{ppm}\left(\mathrm{d},{ }^{3} \mathrm{~J}(\mathrm{H}, \mathrm{H})=4.9 \mathrm{~Hz}, 2 \mathrm{H} ; \beta-\mathrm{H}\right) ;{ }^{13} \mathrm{C}$ NMR $\left(100 \mathrm{MHz}, \mathrm{CDCl}_{3}\right)$ $\delta=14.3,22.8,29.46,29.48,29.75,29.77,29.8,29.9,30.7,30.8,32.0,35.5$, $35.9,38.8,39.0,45.4,46.6,50.4,52.1,65.8,110.7,110.9,116.1,117.4,119.0$, $119.3,125.9,127.8$ (br), 131.0, 131.4, 134.4, 135.6, 136.1, 142.0, 150.3, $166.9 \mathrm{ppm}$; IR (KBr): $\tilde{v}=3316,3120,3053,2952,2923,2852,1704,1605$, 1524, 1475, 1376, 1271, 1177, 1097, 969, 917, 792, $768 \mathrm{~cm}^{-1}$; HRMS (FAB): $m / z$ : calcd for $1116.8034\left(\mathrm{C}_{76} \mathrm{H}_{102} \mathrm{~N}_{5} \mathrm{O}_{2},\left[M^{+}+\mathrm{H}\right]\right)$; found: 1116.8024 .

21H,23H-5-[(4-Aza-tricycle[5.2.1.0 $\left.{ }^{2,6-e n d o}\right]$ dec-8-ene-3,5-dione-4-yl)phenyl]-10,15,20-tris(4-octoxyphenyl)porphyrin (11d): A solution of 10 (43 mg, $0.26 \mathrm{mmol})$ and $5 \mathbf{f}(267 \mathrm{mg}, 0.26 \mathrm{mmol})$ in DMF $(5 \mathrm{~mL})$ was heated at reflux for $18 \mathrm{~h}$. The solvent was removed in vacuo and the residue was purified by column chromatography on silica gel (eluent $\mathrm{CH}_{2} \mathrm{Cl}_{2}$ ) to give the corresponding imide $\mathbf{1 1} \mathbf{d}^{\prime}$ as a purple solid $(201 \mathrm{mg}$, $67 \%$ ). M.p. $>300{ }^{\circ} \mathrm{C} ;{ }^{1} \mathrm{H}$ NMR $\left(300 \mathrm{MHz}, \mathrm{CDCl}_{3}\right): \delta=-2.76(\mathrm{~s}, 2 \mathrm{H}$ $\mathrm{NH}), 0.94\left(\mathrm{t},{ }^{3} \mathrm{~J}(\mathrm{H}, \mathrm{H})=6.6 \mathrm{~Hz}, 9 \mathrm{H} ; \mathrm{CH}_{3}\right), 1.36-1.50\left(\mathrm{~m}, 24 \mathrm{H} ; \mathrm{CH}_{2}\right), 1.61$ (quint., $\left.{ }^{3} J(\mathrm{H}, \mathrm{H})=6.6 \mathrm{~Hz}, 6 \mathrm{H} ; \mathrm{CH}_{2}\right), 1.67\left(\mathrm{~d},{ }^{2} J(\mathrm{H}, \mathrm{H})=9.0 \mathrm{~Hz}, 1 \mathrm{H}\right.$; anti$10-\mathrm{H}), 1.86\left(\mathrm{~d},{ }^{2} J(\mathrm{H}, \mathrm{H})=9.0 \mathrm{~Hz}, 1 \mathrm{H} ;\right.$ syn-10-H), 1.96 (quint., ${ }^{3} J(\mathrm{H}, \mathrm{H})=$ $\left.6.6 \mathrm{~Hz}, 6 \mathrm{H} ; \mathrm{CH}_{2}\right), 3.55$ (brs, $2 \mathrm{H} ; \mathrm{H}$ on norbornene), $3.62(\mathrm{brs}, 2 \mathrm{H} ; \mathrm{H}$ on norbornene), $4.22\left(\mathrm{t},{ }^{3} \mathrm{~J}(\mathrm{H}, \mathrm{H})=6.6 \mathrm{~Hz}, 6 \mathrm{H} ; 3 \times \mathrm{OCH}_{2}\right), 6.43(\mathrm{~s}, 2 \mathrm{H}$; vinyl$\mathrm{H}), 7.25\left(\mathrm{~d},{ }^{3} J(\mathrm{H}, \mathrm{H})=8.1 \mathrm{~Hz}, 6 \mathrm{H} ; \mathrm{ArH}\right), 7.55\left(\mathrm{~d},{ }^{3} J(\mathrm{H}, \mathrm{H})=8.1 \mathrm{~Hz}, 2 \mathrm{H}\right.$ ArH), $8.09\left(\mathrm{~d},{ }^{3} J(\mathrm{H}, \mathrm{H})=8.1 \mathrm{~Hz}, 6 \mathrm{H} ; \operatorname{ArH}\right), 8.26\left(\mathrm{~d},{ }^{3} J(\mathrm{H}, \mathrm{H})=8.1 \mathrm{~Hz}\right.$, $2 \mathrm{H} ; \mathrm{ArH}), 8.87 \mathrm{ppm}(\mathrm{brs}, 8 \mathrm{H} ; \beta-\mathrm{H}) ;{ }^{13} \mathrm{C}$ NMR $\left(75 \mathrm{MHz}, \mathrm{CDCl}_{3}\right): \delta=$ $14.2,22.7,26.2,29.3,29.5,29.7,31.9,45.6,45.9,52.3,68.2,112.7,118.3$ 120.0, 120.3, 124.7, 130.7, 131.0, 131.4, 134.2, 134.8, 135.0, 135.6, 142.4, 158.9, $177.0 \mathrm{ppm}$; IR (KBr): $\tilde{v}=2925,2854,1713,1606,1509,1468,1376$, 1283, 1245, 1174, 1108, 966, 738, $723 \mathrm{~cm}^{-1}$; LRMS (MALDI): $\mathrm{m} / \mathrm{z}(\%)$ $1161.6(100)\left[M^{+}+2 \mathrm{H}\right]$; HRMS (MALDI): $\mathrm{m} / \mathrm{z}$ calcd for $\mathrm{C}_{77} \mathrm{H}_{86} \mathrm{~N}_{5} \mathrm{O}_{5}$ : $1160.6615\left[M^{+}+\mathrm{H}\right]$; found: 1160.6624 .

A solution of imide $11 \mathbf{d}^{\prime}(200 \mathrm{mg}, 0.17 \mathrm{mmol})$ in $\mathrm{CH}_{2} \mathrm{Cl}_{2}(10 \mathrm{~mL})$ was added slowly to a suspension of $\mathrm{LiAlH}_{4}(26 \mathrm{mg}, 0.68 \mathrm{mmol})$ in $\mathrm{Et}_{2} \mathrm{O}$ $(10 \mathrm{~mL})$, and the mixture was stirred at RT for $4 \mathrm{~h}$. Water $(1 \mathrm{~mL})$ and $\mathrm{NaOH}(10 \%, 1 \mathrm{~mL})$ were then added sequentially and the resulting suspension was filtered. The solid was repeatedly triturated with $\mathrm{CH}_{2} \mathrm{Cl}_{2}$ and then filtered. The $\mathrm{CH}_{2} \mathrm{Cl}_{2}$ solution was dried $\left(\mathrm{Na}_{2} \mathrm{SO}_{4}\right)$ and filtered and the filtrate was concentrated in vacuo to give a residue that was purified by column chromatography on silica gel (eluent hexane $/ \mathrm{CH}_{2} \mathrm{Cl}_{2} 1: 1$ ) to give 11 d as a purple solid $(135 \mathrm{mg}, 70 \%)$. M.p. $204-206{ }^{\circ} \mathrm{C} ;{ }^{1} \mathrm{H}$ NMR $\left(300 \mathrm{MHz}, \mathrm{CDCl}_{3}\right): \delta=-2.69(\mathrm{~s}, 2 \mathrm{H} ; \mathrm{NH}), 0.94\left(\mathrm{t},{ }^{3} J(\mathrm{H}, \mathrm{H})=6.6 \mathrm{~Hz}, 9 \mathrm{H}\right.$; $\left.\mathrm{CH}_{3}\right), 1.35-1.71\left(\mathrm{~m}, 32 \mathrm{H} ; \mathrm{CH}_{2}\right.$ on alkyl chain and norbornene), 1.97 (quint., $\left.{ }^{3} \mathrm{~J}(\mathrm{H}, \mathrm{H})=6.6 \mathrm{~Hz}, 6 \mathrm{H} ; \mathrm{CH}_{2}\right), 3.09-3.21(\mathrm{~m}, 6 \mathrm{H} ; \mathrm{H}$ on norbornene), $3.45-3.51(\mathrm{~m}, 2 \mathrm{H} ; \mathrm{H}$ on norbornene $), 4.22\left(\mathrm{t},{ }^{3} \mathrm{~J}(\mathrm{H}, \mathrm{H})=6.6 \mathrm{~Hz}\right.$ $\left.6 \mathrm{H} ; \mathrm{OCH}_{2}\right), 6.34\left(\mathrm{t},{ }^{3} \mathrm{~J}(\mathrm{H}, \mathrm{H})=1.5 \mathrm{~Hz}, 2 \mathrm{H}\right.$; alkenyl $\mathrm{H}$ on norbornene $)$ $6.78\left(\mathrm{~d},{ }^{3} J(\mathrm{H}, \mathrm{H})=8.4 \mathrm{~Hz}, 2 \mathrm{H} ; \operatorname{ArH}\right), 7.25\left(\mathrm{~d},{ }^{3} J(\mathrm{H}, \mathrm{H})=8.4 \mathrm{~Hz}, 6 \mathrm{H}\right.$; $\operatorname{ArH}), 8.01\left(\mathrm{~d},{ }^{3} J(\mathrm{H}, \mathrm{H})=8.4 \mathrm{~Hz}, 2 \mathrm{H} ; \operatorname{ArH}\right), 8.10\left(\mathrm{~d},{ }^{3} \mathrm{~J}(\mathrm{H}, \mathrm{H})=8.4 \mathrm{~Hz}\right.$ $2 \mathrm{H} ; \mathrm{ArH}), 8.83\left(\mathrm{~d},{ }^{3} \mathrm{~J}(\mathrm{H}, \mathrm{H})=4.8 \mathrm{~Hz}, 2 \mathrm{H} ; \beta-\mathrm{H}\right), 8.84(\mathrm{~s}, 4 \mathrm{H} ; \beta-\mathrm{H})$ $8.93 \mathrm{ppm}\left(\mathrm{d},{ }^{3} J(\mathrm{H}, \mathrm{H})=4.8 \mathrm{~Hz}, 2 \mathrm{H} ; \beta-\mathrm{H}\right) ;{ }^{13} \mathrm{C} \mathrm{NMR}\left(75 \mathrm{MHz}, \mathrm{CDCl}_{3}\right)$ $\delta=14.1,22.7,26.3,29.4,29.5,29.7,31.9,45.8,46 ., 51.0,52.3,68.4,110.4$ $112.7,119.3,119.6,121.5,129.4,130.8,130.8,130.9,131.0,131.2,131.2$, $131.3,134.6,134.7,135.6,135.9,136.1,147.2,158.9 \mathrm{ppm}$; IR (KBr): $\tilde{v}=$ 2924, 2853, 1607, 1509,1469, 1367, 1284, 1244, 1174, 965, 803, $738 \mathrm{~cm}^{-1}$, LRMS (MALDI): $m / z$ (\%): $1067.1(100)\left[M^{+}-\mathrm{C}_{4} \mathrm{H}_{3} \mathrm{~N}^{2-}\right], 1132.1$ (6) $\left[M^{+}\right], 1133.1(10)\left[M^{+}+\mathrm{H}\right], 1134.1(10)\left[M^{+}+2 \mathrm{H}\right]$; HRMS (MALDI) $\mathrm{m} / \mathrm{z}$ calcd for $\left.\mathrm{C}_{77} \mathrm{H}_{90} \mathrm{~N}_{5} \mathrm{O}_{3}: 1132.7015\left[M^{+}+\mathrm{H}\right]\right)$; found: 1132.7038 
Compound 11e: Compound $\mathbf{8}$ in $\mathrm{CH}_{2} \mathrm{Cl}_{2}(10 \mathrm{~mL})$ was added dropwise to a mixture of 5e $(507 \mathrm{mg}, 0.5 \mathrm{mmol})$ and DMAP $(0.75 \mathrm{~mol}, 92 \mathrm{mg})$ in $\mathrm{CH}_{2} \mathrm{Cl}_{2}(10 \mathrm{~mL})$ at $0{ }^{\circ} \mathrm{C}$ under argon. The mixture was allowed to warm to RT and was stirred for an additional $24 \mathrm{~h}$. After removal of the solvent in vacuo, the residue was purified by column chromatography on silica gel (eluent $\mathrm{CH}_{2} \mathrm{Cl}_{2} /$ hexane $3: 1$ ) to give 11e as a purple solid (594 mg, $95 \%$ ). M.p. $229-231^{\circ} \mathrm{C} ;{ }^{1} \mathrm{H} \mathrm{NMR}\left(300 \mathrm{MHz}, \mathrm{CDCl}_{3}\right): \delta=-2.76$ (s, $2 \mathrm{H}$; $\mathrm{NH}), 0.94\left(\mathrm{t},{ }^{3} \mathrm{~J}(\mathrm{H}, \mathrm{H})=6.6 \mathrm{~Hz}, 9 \mathrm{H} ; \mathrm{CH}_{3}\right), 1.36-1.67\left(\mathrm{~m}, 32 \mathrm{H} ; \mathrm{CH}_{2}\right), 1.99$ (quint., $\left.{ }^{3} \mathrm{~J}(\mathrm{H}, \mathrm{H})=6.6 \mathrm{~Hz}, 6 \mathrm{H} ; \mathrm{CH}_{2}\right), 3.03-3.07(\mathrm{~m}, 4 \mathrm{H}), 3.12-3.15(\mathrm{~m}$, $2 \mathrm{H}), 3.35-3.41(\mathrm{~m}, 2 \mathrm{H}), 4.25\left(\mathrm{t},{ }^{3} \mathrm{~J}(\mathrm{H}, \mathrm{H})=6.6 \mathrm{~Hz}, 6 \mathrm{H} ; 3 \times \mathrm{OCH}_{2}\right), 6.22(\mathrm{~s}$, $2 \mathrm{H}), 6.52\left(\mathrm{~d},{ }^{3} J(\mathrm{H}, \mathrm{H})=9.0 \mathrm{~Hz}, 2 \mathrm{H}\right), 7.27\left(\mathrm{~d},{ }^{3} J(\mathrm{H}, \mathrm{H})=9.0 \mathrm{~Hz}, 6 \mathrm{H}\right), 7.59$ $\left(\mathrm{d},{ }^{3} J(\mathrm{H}, \mathrm{H})=9.0 \mathrm{~Hz}, 2 \mathrm{H}\right), 8.11\left(\mathrm{~d},{ }^{3} J(\mathrm{H}, \mathrm{H})=9.0 \mathrm{~Hz}, 6 \mathrm{H}\right), 8.18\left(\mathrm{~d},{ }^{3} J-\right.$ $(\mathrm{H}, \mathrm{H})=9.0 \mathrm{~Hz}, 2 \mathrm{H}), 8.23\left(\mathrm{~d},{ }^{3} J(\mathrm{H}, \mathrm{H})=9.0 \mathrm{~Hz}, 2 \mathrm{H}\right), 8.87(\mathrm{~s}, 4 \mathrm{H}, \beta-\mathrm{H})$, $8.89\left(\mathrm{~d},{ }^{3} J(\mathrm{H}, \mathrm{H})=5.1 \mathrm{~Hz}, 2 \mathrm{H}, \beta-\mathrm{H}\right), 8.90 \mathrm{ppm}\left(\mathrm{d},{ }^{3} J(\mathrm{H}, \mathrm{H})=5.1 \mathrm{~Hz}, 2 \mathrm{H}\right.$, $\beta-\mathrm{H}) ;{ }^{13} \mathrm{C} \mathrm{NMR}\left(75 \mathrm{MHz}, \mathrm{CDCl}_{3}\right): \delta=14.2,22.7,26.2,29.3,29.5,31.9$, $45.4,46.6,50.5,52.1,68.3,111.1,112.7,115.2,118.9,119.9,120.1,120.2$, 131.0 (br), 132.1, 134.3, 134.4, 135.3, 135.6, 135.8, 139.3, 150.8, 151.3, 158.9, $165.8 \mathrm{ppm}$; IR (KBr): $\tilde{v}=2924,2851,1722,1604,1558,1506,1471$, $1379,1277,1244,1175,1057,966,802,720 \mathrm{~cm}^{-1}$; LRMS (MALDI): $\mathrm{m} / z$ (\%): $1252.7(100)\left[M^{+}+\mathrm{H}\right]$; HRMS (MALDI): $\mathrm{m} / z$ calcd for $\mathrm{C}_{84} \mathrm{H}_{94} \mathrm{~N}_{5} \mathrm{O}_{5}$ : $1252.7222\left[M^{+}+\mathrm{H}\right]$; found: 1252.7250 ; elemental analysis calcd $(\%)$ for $\mathrm{C}_{84} \mathrm{H}_{94} \mathrm{~N}_{5} \mathrm{O}_{5}$ : C 80.54, $\mathrm{H} 7.48$, N 5.59; found: C 80.07, H 7.51, N 5.11.

Compound $11 \mathrm{f}$ : A mixture of $\mathbf{9 b}(250 \mathrm{mg}, 0.8 \mathrm{mmol})$ and oxalyl chloride $(0.75 \mathrm{~mL}, 8 \mathrm{mmol})$ in $\mathrm{CH}_{2} \mathrm{Cl}_{2}(10 \mathrm{~mL})$ was stirred at $\mathrm{RT}$ for $3 \mathrm{~h}$ and dried in vacuo to give $\mathbf{9 c}$, which was used for the next reaction without further purification. A mixture of $5 \mathbf{e}(507 \mathrm{mg}, 0.5 \mathrm{mmol})$ DMAP (96 mg, $0.8 \mathrm{~mol})$ and $9 \mathrm{c}$ in $\mathrm{CH}_{2} \mathrm{Cl}_{2}(20 \mathrm{~mL})$ was stirred at RT for $24 \mathrm{~h}$. The solvent was removed in vacuo and the residue was purified by column chromatography on silica gel (eluent hexane/ $\left.\mathrm{CH}_{2} \mathrm{Cl}_{2} 3: 7\right)$ to give $\mathbf{1 1} \mathbf{f}(622 \mathrm{mg}$, $95 \%$ ). M.p. $202-204^{\circ} \mathrm{C} ;{ }^{1} \mathrm{H} \mathrm{NMR}\left(300 \mathrm{MHz}, \mathrm{CDCl}_{3}\right): \delta=-2.78(\mathrm{~s}, 2 \mathrm{H}$; $\mathrm{NH}), 0.94\left(\mathrm{t},{ }^{3} J(\mathrm{H}, \mathrm{H})=6.6 \mathrm{~Hz}, 9 \mathrm{H} ; \mathrm{CH}_{3}\right), 1.36-1.67\left(\mathrm{~m}, 32 \mathrm{H} ; \mathrm{CH}_{2}\right), 1.98$ (quint., $\left.{ }^{3} \mathrm{~J}(\mathrm{H}, \mathrm{H})=6.6 \mathrm{~Hz}, 6 \mathrm{H} ; \mathrm{CH}_{2}\right), 2.97-3.00(\mathrm{~m}, 4 \mathrm{H} ; \mathrm{H}$ on norbornene), 3.08-3.11 (m, 2 H; $\mathrm{H}$ on norbornene), 3.29-3.35 (m, $2 \mathrm{H}$; $\mathrm{H}$ on norbornene), $4.24\left(\mathrm{t},{ }^{3} J(\mathrm{H}, \mathrm{H})=6.6 \mathrm{~Hz}, 6 \mathrm{H} ; 3 \times \mathrm{OCH}_{2}\right), 5.20 \quad(\mathrm{~s}, \quad 2 \mathrm{H}$; $\left.\mathrm{OCH}_{2} \mathrm{CO}\right), 6.18$ (s, 2H; vinyl-H), $6.44\left(\mathrm{~d},{ }^{3} J(\mathrm{H}, \mathrm{H})=8.7 \mathrm{~Hz}, 2 \mathrm{H} ; \mathrm{ArH}\right)$, $7.26\left(\mathrm{~d},{ }^{3} J(\mathrm{H}, \mathrm{H})=8.4 \mathrm{~Hz}, 6 \mathrm{H} ; \operatorname{ArH}\right), 7.54\left(\mathrm{~d},{ }^{3} J(\mathrm{H}, \mathrm{H})=8.7 \mathrm{~Hz}, 2 \mathrm{H}\right.$; $\operatorname{ArH}), 8.03\left(\mathrm{~d},{ }^{3} J(\mathrm{H}, \mathrm{H})=8.7 \mathrm{~Hz}, 2 \mathrm{H} ; \mathrm{ArH}\right), 8.09(\mathrm{~d}, J=8.4 \mathrm{~Hz}, 6 \mathrm{H}$; ArH $), 8.21$ (d, $J=8.7 \mathrm{~Hz}, 2 \mathrm{H} ; \operatorname{ArH}), 8.81-8.88 \mathrm{ppm}(\mathrm{m}, 8 \mathrm{H}, \beta-\mathrm{H})$; ${ }^{13} \mathrm{C}$ NMR $\left(100 \mathrm{MHz}, \mathrm{CDCl}_{3}\right): \delta=14.2,22.7,26.2,29.3,29.46,29.47,31.9$, $45.3,46.5,50.3,52.0,60.9,68.2,111.0,112.6,112.7,114.8,118.4,119.6$, $119.8,120.0,120.2,131.1,131.8,134.26,134.29,134.34,135.3,135.5,135.6$, $135.7,140.1,150.1,150.7,158.9,166.5,167.3 \mathrm{ppm}$; IR $(\mathrm{KBr}): \tilde{v}=2926$, 2853, 1745, 1712, 1605, 1507, 1470, 1378, 1283, 1245, 1169, 1104, 966, 801, 768, $720 \mathrm{~cm}^{-1}$; LRMS (MALDI): $m / z(\%): 1310.7\left[M^{+}+\mathrm{H}\right](81)$; HRMS (MALDI): $\mathrm{m} / \mathrm{z}$ calcd for $\mathrm{C}_{86} \mathrm{H}_{96} \mathrm{~N}_{5} \mathrm{O}_{7}: 1310.7318\left[M^{+}+\mathrm{H}\right]$; found: 1310.7304

Compound 1a: A mixture of 11 a $(700 \mathrm{mg}, 0.63 \mathrm{mmol})$ and zinc acetate $(690 \mathrm{mg}, 3.15 \mathrm{mmol})$ in methanol $(20 \mathrm{~mL})$ and $\mathrm{CH}_{2} \mathrm{Cl}_{2}(40 \mathrm{~mL})$ was stirred at RT in the dark for $3 \mathrm{~h}$ and then washed with $\mathrm{NaHCO}_{3}$ and brine and dried $\left(\mathrm{MgSO}_{4}\right)$. The solvent was removed in vacuo to give a residue that was purified by column chromatography on silica gel (eluent hexane $/ \mathrm{CH}_{2} \mathrm{Cl}_{2} / \mathrm{NEt}_{3}$ 1:1:0.05) to afford 1a $(680 \mathrm{mg}, 92 \%)$. M.p. 136$137^{\circ} \mathrm{C} ;{ }^{1} \mathrm{H}$ NMR $\left(400 \mathrm{MHz}, \mathrm{CDCl}_{3}\right): \delta=0.85-0.90\left(\mathrm{~m}, 9 \mathrm{H} ; \mathrm{CH}_{3}\right), 1.27-$ $1.42\left(\mathrm{~m}, 36 \mathrm{H} ; \mathrm{CH}_{2}\right), 1.46-1.61\left(\mathrm{~m}, 7 \mathrm{H} ; \mathrm{CH}_{2} ; \mathrm{CH}\right.$ on norbornene), 1.65 $\left(\mathrm{d},{ }^{2} \mathrm{~J}(\mathrm{H}, \mathrm{H})=8.0 \mathrm{~Hz}, 1 \mathrm{H} ; \mathrm{CH}\right.$ on norbornene $), 1.72-1.85\left(\mathrm{~m}, 6 \mathrm{H} ; \mathrm{CH}_{2}\right)$, 2.30-2.38 (m, 2H; $\left.\mathrm{CH}_{2}\right), 2.39-2.49\left(\mathrm{~m}, 4 \mathrm{H} ; \mathrm{CH}_{2}\right), 3.00-3.04(\mathrm{~m}, 4 \mathrm{H} ; \mathrm{H}$ on norbornene), 3.09-3.11 (m, 2 H; H on norbornene), 3.30-3.35 (m, 2H; $\mathrm{H}$ on norbornene), $4.52\left(\mathrm{t},{ }^{3} \mathrm{~J}(\mathrm{H}, \mathrm{H})=8.4 \mathrm{~Hz} 2 \mathrm{H} ; \mathrm{CH}_{2}\right), 4.71\left(\mathrm{t},{ }^{3} \mathrm{~J}(\mathrm{H}, \mathrm{H})=\right.$ $\left.7.8 \mathrm{~Hz} 4 \mathrm{H} ; \mathrm{CH}_{2}\right), 6.20\left(\mathrm{~s}, 2 \mathrm{H}\right.$; alkenyl $\mathrm{H}$ on norbornene), $6.49\left(\mathrm{~d},{ }^{3} \mathrm{~J}\right.$ $(\mathrm{H}, \mathrm{H})=8.8 \mathrm{~Hz}, 2 \mathrm{H} ; \mathrm{ArH}), 7.60\left(\mathrm{~d},{ }^{3} J(\mathrm{H}, \mathrm{H})=8.8 \mathrm{~Hz}, 2 \mathrm{H} ; \mathrm{ArH}\right), 8.19(\mathrm{~d}$, $\left.{ }^{3} J(\mathrm{H}, \mathrm{H})=8.8 \mathrm{~Hz}, 2 \mathrm{H} ; \mathrm{ArH}\right), 8.20\left(\mathrm{~d},{ }^{3} J(\mathrm{H}, \mathrm{H})=8.8 \mathrm{~Hz}, 2 \mathrm{H} ; \mathrm{ArH}\right), 8.92$ $\left(\mathrm{d},{ }^{3} J(\mathrm{H}, \mathrm{H})=4.8 \mathrm{~Hz}, 2 \mathrm{H} ; \beta-\mathrm{H}\right), 9.12\left(\mathrm{~d},{ }^{3} J(\mathrm{H}, \mathrm{H})=4.8 \mathrm{~Hz}, 2 \mathrm{H} ; \beta-\mathrm{H}\right), 9.16$ $\left(\mathrm{d},{ }^{3} J(\mathrm{H}, \mathrm{H})=4.8 \mathrm{~Hz}, 2 \mathrm{H} ; \beta-\mathrm{H}\right), 9.37 \mathrm{ppm}(\mathrm{d}, J=4.8 \mathrm{~Hz}, 2 \mathrm{H} ; \beta-\mathrm{H})$; ${ }^{13} \mathrm{C} \mathrm{NMR}\left(100 \mathrm{MHz}, \mathrm{CDCl}_{3}\right): \delta=14.3,22.8,29.5,29.79,29.83,29.88,30.9$, $32.0,35.6,35.7,38.97,39.02,45.5,46.8,50.6,52.2,111.1,115.3,118.0$, $119.8,119.9,128.3,128.5,131.7,132.0,134.9,135.7,140.1,148.4,149.1$, 149.2, 149.4, 150.7, 150.8, $165.6 \mathrm{ppm}$; IR (KBr): $\tilde{v}=3123,3060,2955$, 2923, 2852, 1721, 1605, 1527, 1500, 1468, 1380, 1272, 1205, 1175, 1164,
1059, 1008, $788 \mathrm{~cm}^{-1}$; HRMS (FAB): $m / z$ calcd for $\mathrm{C}_{75} \mathrm{H}_{97} \mathrm{~N}_{5} \mathrm{O}_{2} \mathrm{Zn}$ : $1163.6934\left[\mathrm{M}^{+}\right]$; found: 1163.6943 .

Compound 1b: In a manner similar to that described above for $\mathbf{1 a}$, compound $\mathbf{1 1 b}$ (320 mg, $0.287 \mathrm{mmol}$ ) was converted to $\mathbf{1 b}(301 \mathrm{mg}, 89 \%)$ M.p. $68-69^{\circ} \mathrm{C} ;{ }^{1} \mathrm{H} \mathrm{NMR}\left(400 \mathrm{MHz}, \mathrm{CDCl}_{3}\right): \delta=0.89-0.96\left(\mathrm{~m}, 9 \mathrm{H} ; \mathrm{CH}_{3}\right)$, $1.30-1.45\left(\mathrm{~m}, 36 \mathrm{H} ; \mathrm{CH}_{2}\right), 1.45-1.60\left(\mathrm{~m}, 7 \mathrm{H} ; \mathrm{CH}_{2} ; \mathrm{CH}\right.$ on norbornene), $1.64\left(\mathrm{~d},{ }^{2} \mathrm{~J}(\mathrm{H}, \mathrm{H})=8.4 \mathrm{~Hz}, 1 \mathrm{H} ; \mathrm{CH}\right.$ on norbornene $), 1.70-1.85(\mathrm{~m}, 6 \mathrm{H}$; $\left.\mathrm{CH}_{2}\right), 2.26-2.28\left(\mathrm{~m}, 2 \mathrm{H} ; \mathrm{CH}_{2}\right), 2.41-2.43\left(\mathrm{~m}, 4 \mathrm{H} ; \mathrm{CH}_{2}\right), 2.95-2.99(\mathrm{~m}$, $4 \mathrm{H}$; $\mathrm{H}$ on norbornene), 3.06-3.08 (m, 2H; $\mathrm{H}$ on norbornene), 3.24-3.29 (m, 2H; H on norbornene), 4.35-4.39 (m, 2H; $\left.\mathrm{CH}_{2}\right), 4.60-6.64(\mathrm{~m}, 4 \mathrm{H}$; $\left.\mathrm{CH}_{2}\right), 5.69\left(\mathrm{~s}, 2 \mathrm{H} ; \mathrm{OCH}_{2}\right), 6.19(\mathrm{~s}, 2 \mathrm{H}$; alkenyl $\mathrm{H}$ on norbornene), 6.43 $\left(\mathrm{d},{ }^{3} J(\mathrm{H}, \mathrm{H})=8.8 \mathrm{~Hz}, 2 \mathrm{H} ; \operatorname{ArH}\right), 7.84\left(\mathrm{~d},{ }^{3} J(\mathrm{H}, \mathrm{H})=8.0 \mathrm{~Hz}, 2 \mathrm{H} ; \operatorname{ArH}\right)$, $8.06\left(\mathrm{~d},{ }^{3} J(\mathrm{H}, \mathrm{H})=8.8 \mathrm{~Hz}, 2 \mathrm{H} ; \operatorname{ArH}\right), 8.22\left(\mathrm{~d},{ }^{3} J(\mathrm{H}, \mathrm{H})=8.0 \mathrm{~Hz}, 2 \mathrm{H}\right.$; ArH $), 8.88\left(\mathrm{~d},{ }^{3} J(\mathrm{H}, \mathrm{H})=4.8 \mathrm{~Hz}, 2 \mathrm{H} ; \beta-\mathrm{H}\right), 8.98\left(\mathrm{~d},{ }^{3} J(\mathrm{H}, \mathrm{H})=4.0 \mathrm{~Hz}, 2 \mathrm{H}\right.$; $\beta-\mathrm{H}), 9.03\left(\mathrm{~d},{ }^{3} J(\mathrm{H}, \mathrm{H})=4.8 \mathrm{~Hz}, 2 \mathrm{H} ; \beta-\mathrm{H}\right), 9.33 \mathrm{ppm}\left(\mathrm{d},{ }^{3} J(\mathrm{H}, \mathrm{H})=5.2 \mathrm{~Hz}\right.$, $2 \mathrm{H} ; \beta-\mathrm{H}) ;{ }^{13} \mathrm{C}$ NMR $\left(100 \mathrm{MHz}, \mathrm{CDCl}_{3}\right): \delta=14.6,23.1,29.7,30.0,30.1$, $31.1,32.3,35.7,39.1,39.2,45.6,46.8,50.6,52.3,65.9,110.7,115.9,118.0$, $119.36,119.43,125.5,127.3,127.9,128.1,131.1,131.3,134.0,135.35$, $135.41,142.3,147.8,148.4,148.6,148.9,149.9,166.5 \mathrm{ppm}$; IR (KBr): $\tilde{v}=$ 3063, 2952, 2923, 2852, 1704, 1604, 1523, 1466, 1375, 1271, 1177, 1096, $1008,788 \mathrm{~cm}^{-1}$; HRMS (ESI): $\mathrm{m} / z$ calcd for $\mathrm{C}_{76} \mathrm{H}_{99} \mathrm{~N}_{5} \mathrm{O}_{2} \mathrm{Zn}: 1177.7090$ $\left[\mathrm{M}^{+}\right]$; found: 1177.7075 .

Compound 1c: In a manner similar to that described for the preparation of $\mathbf{1 1} \mathbf{a}$, a mixture of $\mathbf{6 b}(500 \mathrm{mg}, 0.51 \mathrm{mmol}), \mathrm{NEt}_{3}(1.0 \mathrm{~mL})$, DMAP (trace amount), and $8(200 \mathrm{mg}, 0.77 \mathrm{mmol}$; freshly prepared from 7) in $\mathrm{CH}_{2} \mathrm{Cl}_{2}(10 \mathrm{~mL})$ was converted to $1 \mathrm{c}(410 \mathrm{mg}, 67 \%)$. M.p. $65-66^{\circ} \mathrm{C}$; ${ }^{1} \mathrm{H}$ NMR $\left(400 \mathrm{MHz}, \mathrm{CDCl}_{3}\right): \delta=0.88-0.93\left(\mathrm{~m}, 9 \mathrm{H} ; \mathrm{CH}_{3}\right), 1.28-1.46(\mathrm{~m}$, $\left.36 \mathrm{H} ; \mathrm{CH}_{2}\right), 1.46-1.66(\mathrm{~m}, 8 \mathrm{H}), 1.77-1.90\left(\mathrm{~m}, 6 \mathrm{H} ; \mathrm{CH}_{2}\right), 2.31-2.54(\mathrm{~m}$, $8 \mathrm{H}), 2.94-2.98(\mathrm{~m}, 4 \mathrm{H}), 3.04-3.07(\mathrm{~m}, 2 \mathrm{H}), 3.18\left(\mathrm{t},{ }^{3} \mathrm{~J}(\mathrm{H}, \mathrm{H})=7.8 \mathrm{~Hz}\right.$, $2 \mathrm{H}), 3.24-3.29(\mathrm{~m}, 2 \mathrm{H}), 4.53-4.56(\mathrm{~m}, 4 \mathrm{H}), 4.74\left(\mathrm{t},{ }^{3} J(\mathrm{H}, \mathrm{H})=7.8 \mathrm{~Hz}\right.$, $4 \mathrm{H}), 6.16(\mathrm{~s}, 2 \mathrm{H}), 6.42\left(\mathrm{~d},{ }^{3} J(\mathrm{H}, \mathrm{H})=8.8 \mathrm{~Hz}, 2 \mathrm{H}\right), 7.62\left(\mathrm{~d},{ }^{3} J(\mathrm{H}, \mathrm{H})=\right.$ $7.8 \mathrm{~Hz}, 2 \mathrm{H}), 7.98\left(\mathrm{~d},{ }^{3} J(\mathrm{H}, \mathrm{H})=8.8 \mathrm{~Hz}, 2 \mathrm{H}\right), 8.12\left(\mathrm{~d},{ }^{3} J(\mathrm{H}, \mathrm{H})=7.8 \mathrm{~Hz}\right.$, $2 \mathrm{H}), 8.90\left(\mathrm{~d},{ }^{3} J(\mathrm{H}, \mathrm{H})=5.0 \mathrm{~Hz}, 2 \mathrm{H}\right), 9.16\left(\mathrm{~d},{ }^{3} J(\mathrm{H}, \mathrm{H})=4.4 \mathrm{~Hz}, 2 \mathrm{H}\right), 9.20$ $\left(\mathrm{d},{ }^{3} \mathrm{~J}(\mathrm{H}, \mathrm{H})=4.4 \mathrm{~Hz}, 2 \mathrm{H}\right), 9.38 \mathrm{ppm}\left(\mathrm{d},{ }^{3} \mathrm{~J}(\mathrm{H}, \mathrm{H})=5.0 \mathrm{~Hz}, 2 \mathrm{H}\right) ;{ }^{13} \mathrm{C}$ NMR $\left(100 \mathrm{MHz}, \mathrm{CDCl}_{3}\right): \delta=14.1,22.7,29.4,29.69,29.75,29.77,30.66,30.74$, $30.80,31.9,32.5,35.0,35.3,38.6,38.8,45.3,46.5,50.4,52.3,63.6,110.9$, $116.6,118.7,119.3,119.4,126.6,127.8,128.0,128.3,131.2,131.7,134.6$, $135.7,140.4,140.9,148.0,148.7,149.3,150.4,167.2 \mathrm{ppm}$; IR $(\mathrm{KBr}): \tilde{v}=$ 3120, 3063, 3022, 2952, 2923, 2852, 1704, 1605, 1524, 1467, 1377, 1274, 1179, 1104, 1077, 1008, 788, 769, $713 \mathrm{~cm}^{-1}$; HRMS (FAB): $\mathrm{m} / \mathrm{z}$ calcd for $\mathrm{C}_{78} \mathrm{H}_{103} \mathrm{~N}_{5} \mathrm{O}_{2} \mathrm{Zn}: 1205.7403\left[M^{+}+\mathrm{H}\right]$; found: 1205.7410 .

Compound 1d: In a manner similar to that described for 1a, compound 11d $(113 \mathrm{mg}, 0.1 \mathrm{mmol})$ was converted to $1 \mathbf{d}(113 \mathrm{mg}, 95 \%)$. M.p. $200-$ $202{ }^{\circ} \mathrm{C} ;{ }^{1} \mathrm{H}$ NMR $\left(300 \mathrm{MHz}, \mathrm{CDCl}_{3}\right): \delta=0.94\left(\mathrm{t},{ }^{3} \mathrm{~J}(\mathrm{H}, \mathrm{H})=6.6 \mathrm{~Hz}, 9 \mathrm{H}\right.$; $\left.\mathrm{CH}_{3}\right), 1.36-1.72(\mathrm{~m}, 32 \mathrm{H}), 1.97$ (quint., ${ }^{3} \mathrm{~J}(\mathrm{H}, \mathrm{H})=6.6 \mathrm{~Hz}, 6 \mathrm{H} ; \mathrm{CH}_{2}$ ), 3.07-3.24 (m, 6H), 3.46-3.51 (m, 2H), $4.23\left(\mathrm{t},{ }^{3} J(\mathrm{H}, \mathrm{H})=6.6 \mathrm{~Hz}, 6 \mathrm{H}\right.$; $\left.\mathrm{OCH}_{2}\right), 6.35(\mathrm{~s}, 2 \mathrm{H}), 6.78\left(\mathrm{~d},{ }^{3} J(\mathrm{H}, \mathrm{H})=8.4 \mathrm{~Hz}, 2 \mathrm{H}\right), 7.25\left(\mathrm{~d},{ }^{3} J(\mathrm{H}, \mathrm{H})=\right.$ $8.4 \mathrm{~Hz}, 6 \mathrm{H}), 8.01\left(\mathrm{~d},{ }^{3} J(\mathrm{H}, \mathrm{H})=8.4 \mathrm{~Hz}, 2 \mathrm{H}\right), 8.10\left(\mathrm{~d},{ }^{3} J(\mathrm{H}, \mathrm{H})=8.4 \mathrm{~Hz}\right.$, $6 \mathrm{H}), 8.94\left(\mathrm{~d},{ }^{3} \mathrm{~J}(\mathrm{H}, \mathrm{H})=4.8 \mathrm{~Hz}, 2 \mathrm{H} ; \beta-\mathrm{H}\right), 8.95(\mathrm{~s}, 4 \mathrm{H} ; \beta-\mathrm{H}), 9.05 \mathrm{ppm}(\mathrm{d}$, $J=4.8 \mathrm{~Hz}, 2 \mathrm{H} ; \beta-\mathrm{H}) ;{ }^{13} \mathrm{C} \mathrm{NMR}\left(75 \mathrm{MHz}, \mathrm{CDCl}_{3}\right): \delta=14.2,22.7,26.3$, 29.4, 29.6, 29.7, 31.9, 45.8, 46.7, 51.0, 52.4, 68.4, 110.3, 112.6, 120.4, 120.7, $122.4,130.0,131.6,131.7,131.8,132.3,135.2,135.4,135.6,136.1,147.1$, $150.36,150.44,150.5,151.0,158.8$ ppm; IR (KBr): $\delta=2924,2853,1606$, 1573, 1524, 1509, 1489, 1468, 1374, 1338, 1281, 1245, 1173, 996, 796, $724 \mathrm{~cm}^{-1}$; LRMS (MALDI): $m / z$ (\%): 1127.3 (100) $\left[M^{+}-\mathrm{Zn}-2 \mathrm{H}\right]$, 1128.3 (90) $\left[M^{+}-\mathrm{Zn}-\mathrm{H}\right], \begin{array}{lllll}1129.3 & (88) & {\left[M^{+}-\mathrm{Zn}\right],} & 1179.4 & (20)\end{array}\left[M^{+}\right.$ $\left.-\mathrm{Zn}+\mathrm{CH}_{3} \mathrm{OH}+\mathrm{H}_{2} \mathrm{O}\right], 1193.3(18)\left[M^{+}\left(\mathrm{Zn}^{64}\right)\right], 1195.3(16)\left[M^{+}\left({ }^{66} \mathrm{Zn}\right)\right]$, 1197.3 (14) $\left[M^{+}\left({ }^{68} \mathrm{Zn}\right)\right] ;$ HRMS (MALDI): $\mathrm{m} / \mathrm{z}$ calcd for $\mathrm{C}_{77} \mathrm{H}_{87} \mathrm{~N}_{5} \mathrm{O}_{3}{ }^{64} \mathrm{Zn:}$ 1193.6092; found: 1193.6095 .

Compound 1e: In a manner similar to that described for $\mathbf{1 a}$, compound 11 e $(500 \mathrm{mg}, 0.4 \mathrm{mmol})$ was converted to $\mathbf{1 e}(510 \mathrm{mg}, 97 \%)$. M.p. $226-$ $228^{\circ} \mathrm{C} ;{ }^{1} \mathrm{H} \mathrm{NMR}\left(300 \mathrm{MHz}, \mathrm{CDCl}_{3}\right): \delta=0.93\left(\mathrm{t},{ }^{3} \mathrm{~J}(\mathrm{H}, \mathrm{H})=6.6 \mathrm{~Hz}, 9 \mathrm{H}\right.$; $\left.\mathrm{CH}_{3}\right), 1.35-1.63\left(\mathrm{~m}, 32 \mathrm{H} ; \mathrm{CH}_{2}\right), 1.95$ (quint., ${ }^{3} J(\mathrm{H}, \mathrm{H})=6.6 \mathrm{~Hz}, 6 \mathrm{H}$; $\left.\mathrm{CH}_{2}\right), 2.93-3.05(\mathrm{~m}, 6 \mathrm{H}), 3.20-3.26(\mathrm{~m}, 2 \mathrm{H}), 4.19\left(\mathrm{t},{ }^{3} \mathrm{~J}(\mathrm{H}, \mathrm{H})=6.6 \mathrm{~Hz}\right.$, $\left.2 \mathrm{H} ; \mathrm{OCH}_{2}\right), 4.20\left(\mathrm{t},{ }^{3} J(\mathrm{H}, \mathrm{H})=6.6 \mathrm{~Hz}, 4 \mathrm{H} ; 2 \times \mathrm{OCH}_{2}\right), 6.16(\mathrm{~s}, 2 \mathrm{H}), 6.41$ $\left(\mathrm{d},{ }^{3} J(\mathrm{H}, \mathrm{H})=9.0 \mathrm{~Hz}, 2 \mathrm{H}\right), 7.22\left(\mathrm{~d},{ }^{3} J(\mathrm{H}, \mathrm{H})=8.4 \mathrm{~Hz}, 2 \mathrm{H}\right), 7.23(\mathrm{~d}, J=$ $8.4 \mathrm{~Hz}, 4 \mathrm{H}), 7.57$ (d, $J=8.4 \mathrm{~Hz}, 2 \mathrm{H}), 8.10\left(\mathrm{~d},{ }^{3} J(\mathrm{H}, \mathrm{H})=8.4 \mathrm{~Hz}, 6 \mathrm{H}\right), 8.12$ $\left(\mathrm{d},{ }^{3} J(\mathrm{H}, \mathrm{H})=9.0 \mathrm{~Hz}, 2 \mathrm{H}\right), 8.23\left(\mathrm{~d},{ }^{3} J(\mathrm{H}, \mathrm{H})=8.4 \mathrm{~Hz}, 2 \mathrm{H}\right), 8.98(\mathrm{brs}, 4 \mathrm{H}$; 
$\beta-\mathrm{H}), 8.99\left(\mathrm{~d},{ }^{3} \mathrm{~J}(\mathrm{H}, \mathrm{H})=4.8 \mathrm{~Hz}, 2 \mathrm{H} ; \beta-\mathrm{H}\right), 9.01 \mathrm{ppm}\left(\mathrm{d},{ }^{3} \mathrm{~J}(\mathrm{H}, \mathrm{H})=4.8 \mathrm{~Hz}\right.$, $2 \mathrm{H} ; \beta-\mathrm{H}) ;{ }^{13} \mathrm{C}$ NMR $\left(100 \mathrm{MHz}, \mathrm{CDCl}_{3}\right): \delta=14.1,22.7,26.2,29.3,29.5$, 29.7, 31.9, 45.3, 46.6, 50.5, 52.1, 68.3, 110.8, 111.1, 112.6, 115.3, 119.98, 120.04, 120.9, 121.0, 131.1, 131.8, 131.9, 132.0, 132.1, 135.00, 135.04, 135.1, $135.4,135.8,139.9,150.2,150.48,150.54,150.6,150.8,151.1,158.76$, $158.78,165.8 \mathrm{ppm}$; IR (KBr): $\tilde{v}=2924,2852,1722,1603,1525,1509,1491$, 1472, 1379, 1338, 1273, 1245, 1204, 1174, 1060, 998, 797, $720 \mathrm{~cm}^{-1}$; LRMS (MALDI): $\mathrm{m} / \mathrm{z}$ (\%): $1313.6(60)\left[\mathrm{M}^{+}\right]$; HRMS (MALDI): $\mathrm{m} / \mathrm{z}$ calcd for $\mathrm{C}_{84} \mathrm{H}_{91} \mathrm{~N}_{5} \mathrm{O}_{5} \mathrm{Zn}$ : $1313.6281\left[M^{+}\right]$); found: 1313.6306; elemental analysis calcd (\%) for $\mathrm{C}_{84} \mathrm{H}_{91} \mathrm{~N}_{5} \mathrm{O}_{5} \mathrm{Zn}$ : C 76.66, H 6.97, N 5.32; found: C 75.86, H 7.32, N 4.93 .

Compound 1f: In a manner similar to that described for 1a, compound $11 \mathrm{f}(131 \mathrm{mg}, 0.1 \mathrm{mmol})$ was converted to $1 \mathbf{f}(115 \mathrm{mg}, 88 \%)$. M.p. 199 $201{ }^{\circ} \mathrm{C} ;{ }^{1} \mathrm{H}$ NMR $\left(300 \mathrm{MHz}, \mathrm{CDCl}_{3}\right): \delta=0.94\left(\mathrm{t},{ }^{3} J(\mathrm{H}, \mathrm{H})=6.6 \mathrm{~Hz}, 9 \mathrm{H}\right.$; $\left.\mathrm{CH}_{3}\right), 1.36-1.60\left(\mathrm{~m}, 32 \mathrm{H} ; \mathrm{CH}_{2}\right), 1.96$ (quint., ${ }^{3} J(\mathrm{H}, \mathrm{H})=6.6 \mathrm{~Hz}, 6 \mathrm{H}$; $\left.\mathrm{CH}_{2}\right), 2.92-2.98(\mathrm{~m}, 4 \mathrm{H} ; \mathrm{H}$ on norbornene), 3.04-3.08 $(\mathrm{m}, 2 \mathrm{H} ; \mathrm{H}$ on norbornene), 3.21-3.27 (m, 2H; H on norbornene), $4.21\left(\mathrm{t},{ }^{3} \mathrm{~J}(\mathrm{H}, \mathrm{H})=6.6 \mathrm{~Hz}\right.$, $\left.6 \mathrm{H} ; 3 \times \mathrm{OCH}_{2}\right), 5.07\left(\mathrm{~s}, 2 \mathrm{H} ; \mathrm{OCH}_{2} \mathrm{CO}\right), 6.16\left(\mathrm{~s}, 2 \mathrm{H} ;\right.$ vinyl-H), $6.36\left(\mathrm{~d},{ }^{3} J-\right.$ $(\mathrm{H}, \mathrm{H})=8.7 \mathrm{~Hz}, 2 \mathrm{H} ; \mathrm{ArH}), 7.24\left(\mathrm{~d},{ }^{3} \mathrm{~J}(\mathrm{H}, \mathrm{H})=8.4 \mathrm{~Hz}, 6 \mathrm{H} ; \mathrm{ArH}\right), 7.50(\mathrm{~d}$, $\left.{ }^{3} J(\mathrm{H}, \mathrm{H})=8.7 \mathrm{~Hz}, 2 \mathrm{H} ; \mathrm{ArH}\right), 7.89\left(\mathrm{~d},{ }^{3} J(\mathrm{H}, \mathrm{H})=8.7 \mathrm{~Hz}, 2 \mathrm{H} ; \mathrm{ArH}\right), 8.09$ $\left(\mathrm{d},{ }^{3} \mathrm{~J}(\mathrm{H}, \mathrm{H})=8.4 \mathrm{~Hz}, 6 \mathrm{H} ; \mathrm{ArH}\right), 8.20\left(\mathrm{~d},{ }^{3} J(\mathrm{H}, \mathrm{H})=8.7 \mathrm{~Hz}, 2 \mathrm{H} ; \mathrm{ArH}\right)$, 8.91-8.98 ppm (m, 8H; $\beta-\mathrm{H}) ;{ }^{13} \mathrm{C}$ NMR $\left(100 \mathrm{MHz}, \mathrm{CDCl}_{3}\right): \delta=14.1,22.7$, $26.2,29.3,29.5,31.9,45.3,46.6,50.4,52.1,60.8,68.3,111.0,112.6,114.7$, $118.4,119.4,119.8,121.0,131.6,131.7,131.86,131.92,132.0,132.1,134.96$, 135.0, 135.1, 135.4, 135.8, 140.8, 149.9, 150.0, 150.49, 150.52, 150.6, 150.7, 158.8, 166.4, 167.3 ppm; IR (KBr): $\tilde{v}=2926,2854,1784,1712,1605,1573$, $1525,1509,1492,1473,1379,1339,1282,1245,1172,1105,998,797$, $720 \mathrm{~cm}^{-1}$; LRMS (MALDI): $\mathrm{m} / \mathrm{z}(\%): 1371.6$ (80) $\left[\mathrm{M}^{+}\right]$; HRMS (MALDI): $\mathrm{m} / \mathrm{z}$ calcd for $\mathrm{C}_{86} \mathrm{H}_{93} \mathrm{~N}_{5} \mathrm{O}_{7} \mathrm{Zn}: 1371.6392\left[M^{+}\right]$; found: 1371.6361.

General procedure for the preparation of polymer 2: A solution of 1 $(0.2 \mathrm{mmol})$ and $\left[\mathrm{Ru}(=\mathrm{CHPh}) \mathrm{Cl}_{2}\left(\mathrm{Cy}_{3} \mathrm{P}\right)_{2}\right](8 \mathrm{mg}, 0.001 \mathrm{mmol}, 5 \mathrm{~mol} \%)$ in $\mathrm{CH}_{2} \mathrm{Cl}_{2}(2 \mathrm{~mL})$ was stirred at $\mathrm{RT}$ for $2 \mathrm{~h}$ Under argon. The mixture was quenched with ethyl vinyl ether $(1 \mathrm{~mL})$ and poured into $\mathrm{MeOH}(10 \mathrm{~mL})$. The resulting solid was collected and redissolved in $\mathrm{CH}_{2} \mathrm{Cl}_{2}(1 \mathrm{~mL})$ and reprecipitated by adding $\mathrm{MeOH}(20 \mathrm{~mL})$. This procedure was repeated two to three times and the solid was collected to afford polymer 2.

Polymer 2a: Yield $92 \%$; ${ }^{1} \mathrm{H} \mathrm{NMR}\left(500 \mathrm{MHz}, \mathrm{CDCl}_{3}\right)$ : $\delta=0.7-0.9(\mathrm{br}$, $\left.9 \mathrm{H} ; \mathrm{CH}_{3}\right), 0.9-1.7$ (br, $\left.44 \mathrm{H} ; \mathrm{CH}_{2}\right), 1.7-2.2\left(\mathrm{br}, 6 \mathrm{H} ; \mathrm{CH}_{2}\right), 2.2-2.5(\mathrm{br}$, $\left.6 \mathrm{H} ; \mathrm{CH}_{2}\right), 2.5-3.0(\mathrm{br}, 4 \mathrm{H}), 3.0-3.9(\mathrm{br}, 8 \mathrm{H}), 4.2-4.7(\mathrm{br}, 2 \mathrm{H}), 4.9-5.6$ (br, 2H), 6.1-6.8 (br, 2H), 7.5-7.8 (br, 2H), 7.8-8.1 (br, 2H), 8.1-8.6 (br, $8 \mathrm{H}), 8.7-9.0 \mathrm{ppm}(\mathrm{br}, 2 \mathrm{H}) ;{ }^{13} \mathrm{C} \mathrm{NMR}\left(125 \mathrm{MHz}, \mathrm{CDCl}_{3}\right): \delta=14.1,22.7$, 29.4, 29.8, 30.7, 30.9, 32.0, 34.4, 35.7, 38.7, 44.4, 46.2, 49.6, 111.7, 116.6, $117.3,118.6,119.3,119.9,126.1,127.4,131.0,132.2,135.4,139.8,148.0$, $150.9,151.3,165.6 \mathrm{ppm}$; IR (KBr): $\tilde{v}=3123,2949,2923,2852,1725,1606$, $1557,1527,1500,1479,1466,1379,1270,1205,1177,1165,1061,1009$, 963, 825, $788 \mathrm{~cm}^{-1}$; GPC (THF): $M_{\mathrm{n}}=18865, M_{\mathrm{w}}=20352, \mathrm{PDI}=1.08$.

Polymer $2 \boldsymbol{b}$ : Yield $91 \%$; ${ }^{1} \mathrm{H} \mathrm{NMR}\left(400 \mathrm{MHz}, \mathrm{CDCl}_{3}\right)$ : $\delta=0.7-0.9(\mathrm{br}$, $\left.9 \mathrm{H} ; \mathrm{CH}_{3}\right), 0.9-1.5\left(\mathrm{br}, 42 \mathrm{H} ; \mathrm{CH}_{2}\right), 1.5-1.9\left(\mathrm{br}, 8 \mathrm{H} ; \mathrm{CH}_{2} ; \mathrm{CH}\right.$ on norbornene), 1.9-2.3 (br, 6H; $\left.\mathrm{CH}_{2}\right), 2.3-3.3(\mathrm{br}, 8 \mathrm{H} ; \mathrm{H}$ on norbornene), 3.8-4.5 (br, $\left.8 \mathrm{H} ; \mathrm{CH}_{2}\right), 4.8-5.4\left(\mathrm{br}, 2 \mathrm{H} ; \mathrm{OCH}_{2}\right), 5.4-5.8(\mathrm{br}, 2 \mathrm{H}),, 6.1-6.6(\mathrm{br}$, $2 \mathrm{H}$; ArH), 7.5-7.7 (br, 2H; ArH), 7.7-8.2 (br, 4H; ArH), 8.2-8.9 ppm (br, $8 \mathrm{H} ; \beta-\mathrm{H}) ;{ }^{13} \mathrm{C}$ NMR $\left(100 \mathrm{MHz}, \mathrm{CDCl}_{3}\right): \delta=14.3,22.8,29.5,29.8$, $29.9,30.8,32.0,35.0,38.9,44.3,46.3,49.4,65.9,111.4,117.6,118.9,119.2$, $125.7,127.6,131.3,134.3,135.4,142.5,147.8,148.1,148.5,150.6$, $166.6 \mathrm{ppm}$; IR (KBr): $\tilde{v}=3123,2952,2922,2852,1707,1606,1522,1466$, $1375,1271,1179,1008,788 \mathrm{~cm}^{-1}$; GPC (THF): $M_{\mathrm{n}}=17136, M_{\mathrm{w}}=20556$, $\mathrm{PDI}=1.19$.

Polymer $2 \boldsymbol{c}$ : Yield $90 \%$; ${ }^{1} \mathrm{H}$ NMR $\left(500 \mathrm{MHz}, \mathrm{CDCl}_{3}\right): \delta=0.7-0.9$ (br, 9H), 0.9-1.6 (br, 44H), 1.6-1.8 (br, 6H), 2.1-2.5 (br, 8H), 2.6-3.3 (br, $8 \mathrm{H}), 3.9-4.6$ (br, 8H), 4.7-5.3 (br, 2H), 6.1-6.5 (br, 2H), 7.4-7.6 (br, $2 \mathrm{H}), 7.7-8.1(\mathrm{br}, 4 \mathrm{H}), 8.5-9.1 \mathrm{ppm}(\mathrm{br}, 8 \mathrm{H}, \beta-\mathrm{H}) ;{ }^{13} \mathrm{C}$ NMR $(125 \mathrm{MHz}$, $\left.\mathrm{CDCl}_{3}\right): \delta=14.1,22.7,29.4,29.7,29.8,30.7,31.9,32.4,35.0,38.8,44.3$, $46.2,49.2,63.7,111.4,117.3,118.3,119.1,126.4,127.8,131.3,134.5,140.2$, 140.7, 147.9, 148.4, 148.9, 150.8, $167.0 \mathrm{ppm}$; IR $(\mathrm{KBr}): \tilde{v}=3018,2952$, 2923, 2852, 1705, 1606, 1523, 1466, 1377, 1273, 1180, 1104, 1077, 1007, 964, 788, 768, $707 \mathrm{~cm}^{-1}$; GPC $(\mathrm{THF}): M_{\mathrm{n}}=15322, M_{\mathrm{w}}=17275$, PDI $=$ 1.13
Polymer $2 \boldsymbol{d}$ : Yield $80 \% ;{ }^{1} \mathrm{H}$ NMR $\left(500 \mathrm{MHz}, \mathrm{CDCl}_{3}\right): \delta=0.89$ (brs, $9 \mathrm{H}$; $\mathrm{CH}_{3}$ ), 1.28-2.00 (br m, 38H), 2.20-3.06 (br m, 8H), 3.40-4.23 (br m, $6 \mathrm{H}$, $\mathrm{OCH}_{2}$ ), 4.80-5.60 (br m, 2H), 6.71-7.28 (br m, 8H), 7.71-8.12 (br m, 8H), $8.67-8.96 \mathrm{ppm}$ (brm, $8 \mathrm{H} ; \beta-\mathrm{H}) ;{ }^{13} \mathrm{C}$ NMR $\left(125 \mathrm{MHz}, \mathrm{CDCl}_{3}\right): \delta=14.1$, 22.7, 26.1, 29.3, 29.5, 29.7, 31.8, 44.5, 45.0, 46.2, 50.2 (br), 68.2 (br), 111.4 $112.2,112.3,112.5,120.3,121.8,129.5,131.5,131.8,132.1,135.3,135.4$, 147.4, 150.2, 150.5, 150.7, $158.5 \mathrm{ppm}$; IR (KBr): $\tilde{v}=3033,2922,2852$, 1606, 1572, 1524, 1509, 1467, 1365, 1338, 1245, 1172, 1106, 1066, 996 (s), $846(\mathrm{w}), 797(\mathrm{~s}), 718 \mathrm{~cm}^{-1}(\mathrm{w}) ;$ GPC (THF): $M_{\mathrm{n}}=10550, M_{\mathrm{w}}=14550$, $\mathrm{PDI}=1.38$

Polymer $2 \boldsymbol{e}$ : Yield $92 \% ;{ }^{1} \mathrm{H}$ NMR $\left(400 \mathrm{MHz}, \mathrm{CDCl}_{3}\right): \delta=0.85$ (brs, $9 \mathrm{H}$; $\left.\mathrm{CH}_{3}\right), 1.23-1.98\left(\mathrm{~m}, 38 \mathrm{H} ; \mathrm{CH}_{2}\right), 2.20-3.30(\mathrm{br}, 8 \mathrm{H}), 3.80-4.25(\mathrm{br}, 6 \mathrm{H}$, $\left.\mathrm{OCH}_{2}\right), 5.14$ (brs, 2H), 6.34 (brs, 2H), 6.87-7.26 (m, 6H), 7.57 (brs, 2H), $7.85-8.20(\mathrm{~m}, 10 \mathrm{H}), 8.85 \mathrm{ppm}(\mathrm{brs}, 8 \mathrm{H} ; \beta-\mathrm{H}) ;{ }^{13} \mathrm{C}$ NMR $(100 \mathrm{MHz}$, $\left.\mathrm{CDCl}_{3}\right): \delta=14.2,22.7,26.1,29.3,29.4,31.8,39.8,44.4,46.8,49.2,68.1$, 111.6, 112.5, 116.2, 119.8, 120.8, 131.9, 135.3, 140.1, 150.0, 150.4, 151.0, 158.5, 158.7, $165.5 \mathrm{ppm}$; IR (KBr): $\tilde{v}=2924,2853,1725,1606,1525,1509$, 1491, 1380, 1339, 1265, 1246, 1205, 1175, 1064, 998, 798, 760, $720 \mathrm{~cm}^{-1}$; elemental analysis calcd (\%): C 76.66, H 6.97, N 5.32; found: C 75.37, H 7.14, N 4.90; GPC (THF): $M_{\mathrm{n}}=51437, M_{\mathrm{w}}=81295, \mathrm{PDI}=1.58$

Polymer $2 f$ : Yield $92 \% ;{ }^{1} \mathrm{H}$ NMR $\left(300 \mathrm{MHz}, \mathrm{CDCl}_{3}\right): \delta=0.91$ (brs, $9 \mathrm{H}$; $\left.\mathrm{CH}_{3}\right), 1.32-1.81\left(\mathrm{~m}, 38 \mathrm{H} ; \mathrm{CH}_{2}\right), 2.60-2.98(\mathrm{~m}, 8 \mathrm{H} ; \mathrm{H}$ on norbornene) $3.95-4.13\left(\mathrm{~m}, 6 \mathrm{H} ; 3 \times \mathrm{OCH}_{2}\right), 5.82\left(\mathrm{brs}, 2 \mathrm{H} ; \mathrm{OCH}_{2} \mathrm{CO}\right), 5.19$ (brs, $2 \mathrm{H}$ vinyl-H), 6.25 (brs, 2H; ArH), 7.01-7.17 (m, 6H; ArH), 7.37 (brs, 2H; ArH), 7.67 (brs, 2H; ArH), 7.93-8.05 (m, 8H; ArH), 8.85-8.91 ppm (m, $8 \mathrm{H} ; \beta-\mathrm{H}) ;{ }^{13} \mathrm{C}$ NMR $\left(100 \mathrm{MHz}, \mathrm{CDCl}_{3}\right): \delta=14.1,22.7,26.2,29.3,29.4$ $31.8,35.7,37.4,39.7,44.3,46.2,49.3,60.6,68.2,111.3,112.5,115.3,119.3$ $120.8,121.0,126.0,128.4,131.6,131.9,132.0,134.96,135.0,135.1,135.3$, 135.4, 140.7, 149.9, 150.4, 150.5, 151.1, 158.5, 158.6, 166.0, $167.0 \mathrm{ppm}$; IR $(\mathrm{KBr}): \tilde{v}=2925,2853,1745,1716,1606,1524,1509,1491,1378,1339$ $1281,1245,1204,1172,1105,998,798,798,767,719 \mathrm{~cm}^{-1}$; GPC (THF): $M_{\mathrm{n}}=9500, M_{\mathrm{w}}=11000, \mathrm{PDI}=1.16$

4-Cyclohexyl-4-aza-tricyclo[5.2.1.0 $\left.{ }^{2,6}\right]$ dec-8-ene (12b): Compound $12 \mathrm{a}^{[26]}$ $(0.50 \mathrm{~g}, 2.04 \mathrm{mmol})$ in THF $(20 \mathrm{~mL})$ was added slowly to a slurry of $\mathrm{LiAlH}_{4}(0.33 \mathrm{~g}, 8.68 \mathrm{mmol})$ in THF $(30 \mathrm{~mL})$, and the mixture was refluxed for $4 \mathrm{~h}$. Water $(3 \mathrm{~mL})$ and $\mathrm{NaOH}(10 \%, 1 \mathrm{~mL})$ were then added and the resulting suspension was filtered. The residue was triturated with THF repeatedly and filtered. The organic solution was dried $\left(\mathrm{MgSO}_{4}\right)$ and filtered, then the filtrate was evaporated in vacuo to give $\mathbf{1 2 b}$ as a liquid $(0.41 \mathrm{~g}, 93 \%)$. ${ }^{1} \mathrm{H}$ NMR $\left(400 \mathrm{MHz}, \mathrm{CDCl}_{3}\right): \delta=1.08-1.22(\mathrm{~m}, 6 \mathrm{H})$, $1.58-1.78(\mathrm{~m}, 2 \mathrm{H}), 2.79(\mathrm{~s}, 2 \mathrm{H}), 2.88-2.92(\mathrm{~m}, 2 \mathrm{H}), 3.01-3.05 \mathrm{ppm}(\mathrm{m}$ $3 \mathrm{H}), 6.10(\mathrm{~s}, 2 \mathrm{H}) ;{ }^{13} \mathrm{C} \mathrm{NMR}\left(100 \mathrm{MHz}, \mathrm{CDCl}_{3}\right): \delta=25.2,26.1,31.8,44.7$ 46.1, 53.9, 54.4, 63.6, $137.3 \mathrm{ppm}$; IR (KBr): $\tilde{v}=3059,2958,2929,2853$ 2796, 2772, 1636, 1449, 1346, 1136, 878, 799, 733, 714, 689, $465 \mathrm{~cm}^{-1}$ HRMS (FAB): $m / z$ calcd for $\mathrm{C}_{15} \mathrm{H}_{24} \mathrm{~N}$ : $218.1909\left[M^{+}+\mathrm{H}\right]$; found: 218.1904

Polymer 13: A solution of $\mathbf{1 2 b}(100 \mathrm{mg}, 0.5 \mathrm{mmol})$ and $\left(\mathrm{Cy}_{3} \mathrm{P}\right)_{2} \mathrm{Cl}_{2} \mathrm{Ru}=$ $\mathrm{CHPh}$ (18 mg, 0.05 equiv) in $\mathrm{CH}_{2} \mathrm{Cl}_{2}(5 \mathrm{~mL})$ was stirred at $\mathrm{RT}$ under argon for $30 \mathrm{~min}$, quenched with ethyl vinyl ether $(1 \mathrm{~mL})$, and poured into $\mathrm{MeOH}(20 \mathrm{~mL})$. The solid was collected and dissolved in $\mathrm{CHCl}_{3}$ $(1 \mathrm{~mL})$ and reprecipitated by adding $\mathrm{MeOH}(20 \mathrm{~mL})$. This procedure was repeated three times and the solid was collected to afford $\mathbf{1 3}$ as a grayish solid (81 mg, $81 \%$ ). ${ }^{1} \mathrm{H}$ NMR (400 MHz, $\left.\mathrm{CDCl}_{3}\right): \delta=1.21(\mathrm{br}, 2 \mathrm{H}), 1.40$ $2.60(\mathrm{br}, 12 \mathrm{H}), 2.56-5.91(\mathrm{br}, 7 \mathrm{H}), 5.40$ (br, for cis double bond, $21 \%$ ), $5.48 \mathrm{ppm}$ (br, for trans double bond, $79 \%$ ); ${ }^{13} \mathrm{C} \mathrm{NMR}\left(100 \mathrm{MHz}, \mathrm{CDCl}_{3}\right.$ ): $\delta=25.2,26.2,29.8,32.1,34.7,36.3,40.3,44.8,45.0,45.8,46.6,54.4,63.6$, 63.7, $130.4 \mathrm{ppm}$ and residual signals for end groups. IR (KBr): $\tilde{v}=3040$, 2926, 2851, 2773, 1632, 1448, 1372, 1135, 969, 890, $691 \mathrm{~cm}^{-1}$; End-group analysis showed that polymer $\mathbf{1 3}$ contained 27 repetitive units.

Time-resolved fluorescence experiments: A mode-locked Ti:sapphire laser (wavelength $850 \mathrm{~nm}$, repetition rate $76 \mathrm{MHz}$, pulse width $<200 \mathrm{fs}$ ) was passed through an optical parametric amplifier to produce a $\lambda=$ $425 \mathrm{~nm}$ pulse laser. The fluorescence of the sample was reflected by a grating $\left(150 \mathrm{~g} \mathrm{~mm}^{-1}\right.$, BLZ $\left.500 \mathrm{~nm}\right)$ and detected by an optically triggered streak camera (Hamamatsu C5680) with a time resolution of about 0.3 ps. Each $1 \times 10^{-5} \mathrm{M}$ sample was prepared in $\mathrm{CH}_{2} \mathrm{Cl}_{2}$ and placed in an ultra-micro cuvet with $1 \mathrm{~mm}$ pathlength to maintain simultaneous excita- 
tion. The signal was collected twenty times to decrease signal-to-noise ratio $\left[A_{1} \exp \left(-t / \tau_{1}\right)+A_{2} \exp \left(-t / \tau_{2}\right)\right]$.

\section{Acknowledgements}

We thank the National Science Council, the National Taiwan University, Taipei, and Shanghai Institute of Organic Chemistry, Shanghai, for support.

[1] a) G. McDermott, S. M. Prince, A. A. Freer, A. M. Hawthornthwaite-Lawless, M. Z. Papiz, R. J. Cogdell, N. W. Isaacs, Nature 1995, 374, 517-521; b) J. Koepke, X. Hu, C. Muenke, K. Schulten, H. Michel, Structure 1996, 4, 581-597; c) T. Pullerits, V. Sundstrom, Acc. Chem. Res. 1996, 29, 381-389.

[2] a) Y. Nakamura, N. Aratani, A. Osuka, Chem. Soc. Rev. 2007, 36, 831-845; b) M. R. Wasielewski, J. Org. Chem. 2006, 71, 5051-5066; c) A. Satake, Y. Kobuke, Org. Biomol. Chem. 2007, 5, 1679-1691; d) M.-S. Choi, T. Yamazaki, I. Yamazaki, T. Aida, Angew. Chem. 2004, 116, 152-160; Angew. Chem. Int. Ed. 2004, 43, 150-158; e) D. Kim, A. Osuka, Acc. Chem. Res. 2004, 37, 735-745; f) P. D. Harvey, C. Stern, C. P. Gros, R. Guilard, J. Inorg. Biochem. 2008, 102, 395405; g) P. D. Harvey, C. Stern, C. P. Gros, R. Guilard, Coord. Chem. Rev. 2007, 251, 401-428.

[3] a) I. Abdalmuhdi, C. K. Chang, J. Org. Chem. 1985, 50, 411-413; b) T. Nagata, A. Osuka, K. Maruyama, J. Am. Chem. Soc. 1990, 112, 3054-3059; c) A. Osuka, S. Nakajima, K. Maruyama, J. Am. Chem. Soc. 1992, 114, 7355-7359; d) S. G. Boxer, R. R. Buck, J. Am. Chem. Soc. 1979, 101, 1883-1885.

[4] a) M.-S. Choi, T. Aida, T. Yamazaki, I. Yamazaki, Chem. Eur. J. 2002, 8, 2667-2678; b) W. S. Li, K. S. Kim, D. L. Jiang, H. Tanaka, T. Kawai, J. H. Kwon, D. Kim, T. Aida, J. Am. Chem. Soc. 2006, 128, 10527-10532.

[5] a) M. Endo, M. Fujitsuka, T. Majima, Chem. Eur. J. 2007, 13, 86608666; b) F. X. Redl, M. Lutz, J. Daub, Chem. Eur. J. 2001, 7, 53505358; c) T. Konishi, A. Ikeda, M. Asai, T. Hatano, S. Shinkai, M. Fujitsuka, O. Ito, Y. Tsuchiya, J. i. Kikuchi, J. Phys. Chem. B 2003, 107, 11261-11266; d) F. Takei, H. Hayashi, K. Onitsuka, N. Kobayashi, S. Takahashi, Angew. Chem. 2001, 113, 4216-4218; Angew. Chem. Int. Ed. 2001, 40, 4092-4094; e) P. A. J. de Witte, M. Castriciano, J. J. L. M. Cornelissen, L. M. Scolaro, R. J. M. Nolte, A. E. Rowan, Chem. Eur. J. 2003, 9, 1775-1781; f) F. Takei, D. Kodama, S. Nakamura, K. Onitsuka, S. Takahashi, J. Polym. Sci. Part A: Polym. Chem. 2006, 44, 585-595; g) M. Fujitsuka, A. Okada, S. Tojo, F. Takei, K. Onitsuka, S. Takahashi, T. Majima, J. Phys. Chem. B 2004, 108, 11935-11941.

[6] a) M. Sykora, K. A. Maxwell, J. M. DeSimone, T. J. Meyer, Proc. Natl. Acad. Sci. USA 2000, 97, 7687-7691; b) J. H. Alstrum-Acevedo, M. K. Brennaman, T. J. Meyer, Inorg. Chem. 2005, 44, 68026827.

[7] M. A. Fox, Acc. Chem. Res. 1999, 32, 201-207.
[8] A. J. Myles, B. Gorodetsky, N. R. Branda, Adv. Mater. 2004, 16, 922 925.

[9] a) J. A. Sattigeri, C. W. Shiau, C. C. Hsu, F. F. Yeh, S. Liou, B. Y. Jin, T. Y. Luh, J. Am. Chem. Soc. 1999, 121, 1607-1608; b) W.-Y. Lin, M. G. Murugesh, S. Sudhakar, H.-C. Yang, H.-C. Tai, C.-S. Chang, Y.-H. Liu, Y. Wang, I. W. P. Chen, C.-h. Chen, T.-Y. Luh, Chem. Eur. J. 2006, 12, 324-330; c) W.-Y. Lin, H.-W. Wang, Z.-C. Liu, J. Xu, C.W. Chen, Y.-C. Yang, S.-L. Huang, H.-C. Yang, T.-Y. Luh, Chem. Asian J. 2007, 2, 764-774.

[10] a) H.-C. Yang, S.-Y. Lin, H.-C. Yang, C.-L. Lin, L. Tsai, S.-L. Huang, I. W.-P. Chen, C.-h. Chen, B.-Y. Jin, T.-Y. Luh, Angew. Chem. 2006, 118, 740-744; Angew. Chem. Int. Ed. 2006, 45, 726-730; b) N.-T. Lin, S.-Y. Lin, S.-L. Lee, C.-H. Chen, C.-H. Hsu, L. P. Hwang, Z.-Y. Xie, C.-H. Chen, S.-L. Huang, T.-Y. Luh, Angew. Chem. 2007, 119, 4565-4569; Angew. Chem. Int. Ed. 2007, 46, 4481-4485.

[11] C.-L. Lin, H.-C. Yang, N.-T. Lin, I-J. Hsu, Y. Wang, T.-Y. Luh, Chem. Commun. 2008, 4484-4486.

[12] a) J. S. Lindsey, I. C. Schreiman, H. C. Hsu, P. C. Kearney, A. M. Marguerettaz, J. Org. Chem. 1987, 52, 827-836; b) J. S. Lindsey, R. W. Wagner, J. Org. Chem. 1989, 54, 828-836.

[13] The peaks were slightly sharper at elevated temperatures (e.g., $403 \mathrm{~K}$ ). For a typical example for $\mathbf{2 b}$, see the Supporting Information.

[14] For a review, see: Y. Kobuke, Eur. J. Inorg. Chem. 2006, 2333-2351.

[15] T. S. Balaban, A. Eichhöfer, J.-M. Lehn, Eur. J. Org. Chem. 2000, 4047-4057.

[16] S.-L. Lee, C.-Y. J. Chi, M.-J. Huang, C.-h. Chen, C.-W. Li, K. Pati, R.-S. Liu, J. Am. Chem. Soc. 2008, 130, 10454-10455.

[17] H. L. Anderson, Chem. Commun. 1999, 2323-2330.

[18] A. Osuka, K. Maruyama, J. Am. Chem. Soc. 1988, 110, 4454-4456.

[19] S. Faure, C. Stern, R. Guilard, P. D. Harvey, J. Am. Chem. Soc. 2004, 126, 1253-1261.

[20] M. Kasha, H. R. Rawls, M. A. El-Bayoumi, Pure Appl. Chem. 1965, 11, 371-392.

[21] a) R. E. Overfield, A. Scherz, K. J. Kaufmann, M. R. Wasielewski, J. Am. Chem. Soc. 1983, 105, 4256-4260; b) R. R. Bucks, T. L. Netzel, I. Fujita, S. G. Boxer, J. Phys. Chem. 1982, 86, 1947-1955.

[22] a) M. J. Yuen, L. L. Shipman, J. J. Katz, J. C. Hindman, Photochem Photobiol. 1980, 32, 281-296; b) J. B. Clark, A. L. Smirl, E. W. Van Stryland, H. J. Mackey, B. R. Russell, Chem. Phys. Lett. 1981, 78, 456-460; c) D. R. Lutz, K. A. Nelson, C. R. Gochanour, M. D. Fayer, Chem. Phys. 1981, 58, 325-334.

[23] N. Spreti, L. Brinchi, R. Germani, M. V. Mancini, G, Savelli, Eur. J. Org. Chem. 2006, 4379-4384.

[24] B. A. Gregg, M. A. Fox, A. J. Bard, Tetrahedron 1989, 45, 47074716.

[25] R. F. Kelley, M. J. Tauber, M. R. Wasielewski, J. Am. Chem. Soc 2006, 128, 4779-4791.

[26] K. Diaz, J. Vargas, L. F. D. Castillo, M. A. Tlenkopatchev, M. Aguilar-Vega, Macromol. Chem. Phys. 2005, 206, 2316-2322.

Received: January 22, 2009 Published online: April 22, 2009 